\title{
ANÁLISIS BIBLIOMÉTRICO DE LAS REVISTAS ESPAÑOLAS DE EDUCACIÓN INCLUIDAS EN EL JOURNAL CITATION REPORT. PRODUCCIÓN CIENTÍFICA Y ELEMENTOS CONTROVERTIDOS
}

\author{
Bibliometric analysis of Spanish journals of education \\ indexed in Journal Citation Report. Scientific production \\ and controversial elements
}

Analyse bibliométrique des revues espagnoles d'éducation dans le Journal Citation Report. Production scientifique et éléments controversés

Juan Luis FueNTES*, David LuQuE** y Ernesto LóPez GómeZ****

* Universidad Complutense de Madrid. Facultad de Educación. Departamento de Teoría e Historia de la Educación. C/ Rector Royo Villanova, s/n. 28040 Madrid. Correo-e: juanluis.fuentes@edu.ucm.es

** Universidad Complutense de Madrid. Facultad de Educación. Departamento de Teoría e Historia de la Educación. C/ Rector Royo Villanova, s/n. 28040 Madrid. Correo-e: dluque@edu.ucm.es

**** Centro Universitario Villanueva (adscrito a la Universidad Complutense de Madrid). Área de Educación. Departamento de Pedagogía y Contextos Educativos. C/ Costa Brava, n. ${ }^{\circ}$ 2. 28034 Madrid. Correo-e: elopezg@villanueva.edu

Fecha de recepción: enero de 2012

Fecha de aceptación definitiva: abril de 2012

Biblid [(1130-3743) 24, 1-2012, 183-217] 


\section{RESUMEN}

Este artículo presenta un análisis bibliométrico de las revistas españolas de educación incluidas en 2010 en el Journal Citation Report, a saber, Revista Española de Pedagogía, Infancia y Aprendizaje, Teoría de la Educación. Revista Interuniversitaria, Revista de Psicodidáctica y Comunicar: Revista Científica de Comunicación y Educación. Se atiende a dos aspectos fundamentales. A los datos generales que nos ofrecen los artículos publicados por un lado y, por el otro, a los elementos controvertidos que más discusión suscitan entre los expertos en revistas científicas: el nivel de endogamia, la financiación de las investigaciones y el grado de internacionalización de cada revista. Como conclusión, se ofrecen algunas reflexiones que pueden ofrecer luz sobre la situación actual de algunas de las revistas científicas de educación más relevantes editadas en España.

Palabras clave: estudio bibliométrico, Journal Citation Report, producción científica, endogamia, financiación de la investigación, internacionalización.

\section{SUMMARY}

This article presents a bibliometric analysis of the Spanish journals of education included in 2010 in Journal Citation Report. These journals are Revista Española de Pedagogía, Infancia y Aprendizaje, Teoría de la Educación. Revista Interuniversitaria, Revista de Psicodidáctica and Comunicar: Revista Científica de Comunicación y Educación. Our research focuses on two fundamental elements: first, general data given by the studied journals; second, controversial elements where more arguments arise between experts in scientific journals: the level of inbreeding, the research financing and the degree of internationalization of each journal. Finally, it presents some reflections which can illuminate the current situation of some of the most relevant journals of education in Spain.

Key words: bibliometric research, Journal Citation Report, scientific production, inbreeding, research financing, internationalization.

\section{SOMMAIRE}

Cet article présente une analyse bibliométrique sur les revues espagnoles d'éducation qui sont indexées en 2010 dans le Journal Citation Report, à savoir, Revista Española de Pedagogía, Infancia y Aprendizaje, Teoría de la Educación. Revista Interuniversitaria, Revista de Psicodidáctica et Comunicar: Revista Científica de Comunicación y Educación. Le travail se base sur deux aspects fondamentaux. D’un côté, nous appliquons les données générales offertés par les articles publiés. De l'autre, nous remarquons les éléments controversés qui ont suscité plus de discussion entre les experts dans les revues scientifiques, tels que le niveau d'endogamie, le financement de la recherche et le niveau d'internationalisation de chaque revue. Finalement, nous proposons quelques réflexions qui peuvent montrer la situation actuelle de certaines revues scientifiques d'éducation qui sont parmi les plus importantes publiées en Espagne.

Mots clés: étude bibliométrique, Journal Citation Report, production scientifique, endogamie, financement de la recherche, internationalisation. 


\section{JUSTIFICACIÓN}

El sistema de difusión del conocimiento científico ha cambiado progresivamente de paradigma desde la primera Europa del siglo XVII. Atrás quedaron las exclusivas reuniones de intelectuales donde intercambiar impresiones, la creación de sociedades privilegiadas como la Royal Society of London's Philosophical Transactions (Hamilton y Weiner, 2011) o la French Academy's Journal des Sçavans (Vicentelli, 2009), las disputas por la autoría de los descubrimientos, donde encontramos los casos de Isaac Newton y Leibniz en la década de 1660 con el desarrollo del cálculo, los intercambios epistolares o las ediciones de libros y monográficos restringidas a un ámbito reducido. Sería tras este desarrollo de las comunidades científicas cuando se adoptara como la solución más plausible la edición de revistas de carácter internacional que recogieran artículos con un formato estándar escritos en lengua inglesa (Argüelles, 2008).

Desde entonces y hasta hoy, uno de los principales quehaceres de cualquier investigador que se precie es el de difundir los conocimientos que alcanza al resto de la comunidad científica de manera tal que, aunque el intercambio siga produciéndose en congresos, seminarios o libros, son sobre todo los articles los elementos que con mayor fuerza se han erigido tanto en un proceso de popularización de la cultura científica (Argüelles, 2008) como en las agencias de acreditación para docentes o investigadores universitarios donde la evaluación del profesorado es cada vez más importante (Vázquez, Colom y Sarramona, 1998). Sirva, a modo de ejemplo, la Agencia Nacional de Evaluación de la Calidad y la Acreditación (ANECA) u otras agencias de competencia autonómica (Bellés, 2006).

Como cabía esperar, junto al crecimiento de las publicaciones científicas ha florecido también toda una industria dedicada a evaluar la calidad de los journals con los fines de que los científicos conozcan con mayor precisión cuáles son los mejores destinos si desean que sus escritos lleguen a un número elevado de lectores, sean potencialmente citados por un número también alto de investigadores o sirvan como criterio para la evaluación del profesorado universitario. De entre esta amalgama de agencias y sistemas de evaluación de los journals, el criterio que con más fuerza ha arraigado es el conocido como "factor de impacto", usado por la empresa Thomson Reuters, y dado a conocer a través del Journal Citation Report (JCR). A pesar de las alternativas existentes, que las hay (Aleixandre, ValderramaZurián y González Alcaide, 2007), y de las críticas que se han vertido, que también las hay (Aleixandre, 2010a; Delgado López-Cózar, 2007; Delgado López-Cózar et al., 2005), este método se ha erigido como el más fiable, internacional y sencillo, de tal forma que la pertenencia a la lista que "Thomson Reuters ISI" actualiza anualmente según campos del saber es motivo de orgullo para los directores y editores de las revistas científicas. El ámbito de la investigación educativa en España no es distinto en este punto.

La producción científica educativa española ha crecido de forma considerable en cantidad y calidad en los últimos años donde el número de revistas especializadas 
ha aumentado de forma considerable desde la creación, en 1904, de la Cátedra de Pedagogía Superior en la Facultad de Filosofía y Letras en la Universidad Central de Madrid (Hernández Díaz, 2010; Ruiz Berrio y Vázquez Gómez, 2005) dentro de los estudios de doctorado en filosofía.

No olvidamos, no obstante, el Boletín de la Institución Libre de Enseñanza, que se viene publicando desde 1877, ni el reconocimiento de la labor del Consejo Superior de Investigaciones Científicas que, con la creación en 1941 del Instituto de Pedagogía San José de Calasanz, animó el cultivo de líneas de investigación tales como la Filosofía de la Educación, Bibliografía Pedagógica, Historia de la Pedagogía y Métodos Experimentales aplicados a la educación que se difundirían a través de la Revista Española de Pedagogía, cuyo primer número vería la luz en 1943 (Galino, 2005). Unos años después, en 1948, se fundó la Sociedad Española de Pedagogía por unos jóvenes licenciados que después, con el discurrir de los años, serían eminentes pedagogos con un legado indiscutible, como es el caso de Víctor García Hoz. Bajo el auspicio de esta Sociedad se comenzó a editar la revista Bordón que, junto a la ya citada Revista Española de Pedagogía, han sido referentes en el desarrollo de la pedagogía en España en el último medio siglo (Fundación Santa María, 1994). Obviamente, con el discurrir de la disciplina pedagógica y la necesidad de una mayor difusión de las investigaciones han ido apareciendo, especialmente durante las tres últimas décadas, un amplio número de revistas especializadas difícilmente cuantificables ${ }^{1}$.

Sin embargo, el JCR esperó hasta el año 2007 para inaugurar la presencia española $-\mathrm{y}$ de revistas en lengua castellana- en materia educativa con la Revista Española de Pedagogía, a la que habrían de seguir en los años posteriores, la revista Infancia y Aprendizaje, que se incorporó en 2008, y las revistas Teoría de la Educación. Revista Interuniversitaria, Revista de Psicodidáctica y Comunicar: Revista Científica de Comunicación y Educación, que se incluyeron en $2009^{2-3}$.

1. Para una perspectiva de las revistas educativas españolas puede acudirse al índice bibliométrico IN-RECS, elaborado por la Universidad de Granada, que se ha convertido en una referencia nacional en la evaluación de las revistas científicas en Ciencias Sociales desde mediados de los noventa. Por otro lado, desde 2009, la Red Española de Revistas Científicas de Educación dirigidas por el Dr. García del Dujo viene siendo un canal de comunicación entre algunas de las revistas científicas editadas en España e indexadas en el JCR o evaluadas positivamente por el Repositorio Español de Ciencia y Tecnología (RECYT) de la Fundación Española de Ciencia y Tecnología (FECYT).

2. En junio del 2011 se han incluido cuatro revistas más: Cultura y Educación que, junto a Infancia y Aprendizaje, suman dos publicaciones periódicas editadas por la Fundación Infancia y Aprendizaje; Enseñanza de las Ciencias, editada por la Universidad Autónoma de Barcelona; Porta Linguarum, editada por la Universidad de Granada; y Revista de Educación, editada por el Ministerio de Educación.

3. Dentro del JCR, Revista Española dePedagogía y Teoría delaEducación. Revista Interuniversitaria se encuentran en la categoría Education E Educational Research; Infancia y Aprendizaje y Revista de Psicodidáctica se ubican en la sección "Psychology, Educational"; mientras que Comunicar: Revista 
Los estudios de carácter bibliométrico realizados hasta ahora sobre las revistas educativas españolas analizan publicaciones de manera individual ( $c f r$. GómezGarcía, Ramiro, Ariza y Granados, 2012; Carpallo y Burgos, 2009; Granados, Ariza, Gómez-García y Ramiro, 2011; Zych, 2011; Gutiérrez-Arenas, Maz-Machado e Hidalgo-Ariza, 2010; Pineda, 1985), motivados por algún acontecimiento de relevancia para la revista (García Sánchez, Sánchez Miguel, Arias-Gundín y Del Río, 2002; Sánchez Miguel, García Sánchez y Del Río, 2002) o enfocados a temáticas concretas en una o varias revistas (Flores Buils, Gil Beltrán, Caballer y Martínez Martínez, 2012; Bracho-López et al., 2010). Más cercanos al estudio que aquí presentamos son los trabajos de Fernández Cano (1999, 2011) que analizan la producción científica de los autores españoles en el Social Sciences Citation Index (SSCI), aportando una perspectiva diferente a la de nuestra investigación al referirse a la participación española en publicaciones de 91 revistas, de las cuales sólo 10 son nacionales. Además, aunque atiende a algunas variables similares a las aquí incluidas, debido a que el objeto de estudio es diferente, obtiene resultados diferentes. Asimismo, cabe destacar su recomendación de analizar en investigaciones posteriores otros aspectos importantes como la endogamia, para posibilitar una comprensión más fiable de los resultados (2011, 440).

Así pues, hasta la fecha no existen estudios comparativos que engloben diferentes revistas en torno a un criterio de calidad internacional. Con esta pretensión presentamos nuestro trabajo.

La presente investigación se propone en definitiva conocer la producción científica española en el ámbito educativo durante la primera década del tercer milenio, a través de un estudio bibliométrico que compara la producción científica de las cinco primeras revistas españolas de naturaleza educativa que se incluyeron en el $J C R$, con particular atención a ciertos elementos que pueden considerarse "controvertidos", lo que dejaría finalmente los objetivos específicos en los siguientes:

1. Realizar un estudio bibliométrico general donde se atienda a las siguientes variables:

a) Datos generales por revista.

b) Producción científica.

c) Autoría de los trabajos.

d) Instituciones representadas.

2. Comprobar el grado en que las revistas científicas publican artículos escritos por los miembros de su propio organigrama científico, o por investigadores pertenecientes a la universidad que financia la revista.

Científica de Comunicación y Educación debe buscarse en "Communication", aunque cabe señalar que en 2011 fue incluida también en la categoría específicamente educativa. 
3. Estudiar el porcentaje de artículos financiados, prestando especial atención a las categorías del JCR donde se ubican las revistas estudiadas.

4. Observar el grado de internacionalización de las revistas científicas atendiendo a la procedencia de los autores, idioma de publicación y traducciones publicadas.

\section{MÉTODO}

La bibliometría se define como el conjunto de estudios que tratan de cuantificar el proceso de comunicación escrita, la naturaleza y evolución de las disciplinas científicas y su reflejo en la literatura, mediante el recuento y análisis de diversas características de dicha comunicación (Aleixandre, 2010b). En consecuencia, se realiza un análisis bibliométrico con carácter descriptivo al considerar que es el método más pertinente para lograr los objetivos propuestos.

Nuestra unidad de análisis es el artículo científico publicado en las revistas objeto de estudio excluyendo aquellas introducciones, discusiones, notas del editor y otras secciones que pese a su interés no reúnen los requisitos y parámetros de formato que sí cumplen los artículos científicos.

Se han analizado un total de 1.314 artículos publicados durante la primera década del tercer milenio, en los que colaboran individual o colectivamente 1.779 autores diferentes, en un total de 2.391 participaciones. El motivo de la elección de este periodo de tiempo es que se revela como el más justo tanto para las primeras revistas que entraron en el selectivo índice como para las que entraron más tarde, debido a que todas tienen periodos durante los años 2001-2010 en las que estaban indexadas en él y periodos de tiempo de al menos cinco años en las que no lo estaban, aunque realmente la diferencia desde que entró la Revista Española de Pedagogía y el resto es de tan sólo dos años como máximo.

Por supuesto, existen otros índices que pese a ligeras variantes tienen la misma finalidad que el $J C R$, pero se considera que el ámbito selectivo del pequeño grupo que pertenece a este índice y la diferencia de calidad que marca con respecto a otros es tan notable que en un momento histórico en que las universidades europeas, entre las que por supuesto se cuentan también las españolas, persiguen la excelencia, y si la excelencia es lo que sobresale, se antoja como lo más sensato desde un punto de vista científico escoger un índice similar.

Finalmente, cabe indicar que aunque las revistas objeto de estudio comparten un criterio común de exigencia y calidad expresado en $J C R$, presentan una cierta diversidad -en sus temáticas, estructuras formales y de estilo, extensión de los artículos, composición de los organigramas, periodicidad, números monográficos y secciones incluidas- que, sin embargo, no supone una dificultad significativa para realizar un estudio comparativo entre ellas. 


\section{Resultados}

\subsection{Resultados bibliométricos generales}

\subsubsection{Datos generales}

TABla 1. Datos generales POR REVISTA

\begin{tabular}{|c|c|c|c|c|c|c|}
\hline & General & REP & T. ${ }^{a}$ de la Ed. & I\&A & Psicodidáctica & Comunicar \\
\hline \multirow{2}{*}{ N. ${ }^{\circ}$ de artículos } & 1.314 & 244 & 105 & 300 & 163 & 502 \\
\hline & $100 \%$ & $18,6 \%$ & $8 \%$ & $22,8 \%$ & $12,4 \%$ & $38,25 \%$ \\
\hline $\begin{array}{l}\mathrm{N} .^{\circ} \text { de } \\
\text { ejemplares } \\
\text { de revista }\end{array}$ & 119 & 30 & 12 & 40 & 17 & 20 \\
\hline $\begin{array}{l}\text { Ejemplares } \\
\text { por año }\end{array}$ & & 3 & $1-2$ & 4 & $1-2$ & 2 \\
\hline $\begin{array}{c}\bar{X} \text { de artículos } \\
\text { por ejemplar }\end{array}$ & & 8,13 & 8,75 & 7,5 & 9,59 & 25,1 \\
\hline $\begin{array}{c}\bar{X} \text { de artículos } \\
\text { por año }\end{array}$ & & 24,4 & 10,5 & 30 & 16,3 & 50,2 \\
\hline $\begin{array}{l}\text { Mín. y máx. } \\
\text { por ejemplar }\end{array}$ & & $6-11$ & $6-12$ & $5-14$ & $6-19$ & $20-34$ \\
\hline $\begin{array}{l}\text { Mín. y máx. } \\
\text { por año }\end{array}$ & & $23-26$ & $6-15$ & $25-40$ & $12-24$ & 41-64 \\
\hline $\begin{array}{c}\text { Autores } \\
\text { diferentes }\end{array}$ & $1.779^{*}$ & 382 & 118 & 561 & 261 & 563 \\
\hline $\begin{array}{c}\text { Autores totales } \\
\text { (repetidos) }\end{array}$ & 2.391 & 442 & 162 & 787 & 335 & 665 \\
\hline $\begin{array}{c}\bar{X} \text { Autores } \\
\text { por año }\end{array}$ & & 38,2 & 11,8 & 56,1 & 26,1 & 56,3 \\
\hline
\end{tabular}

* La suma de autores de cada una de las revistas por separado es superior al número total de autores debido a que algunos publican en varias revistas.

\subsubsection{Producción científica}

3.1.2.1. Evolución del número de artículos publicados

TABLA 2. EVOlUCIÓN DEL NÚMERO DE ARTíCUlOS PUBLICADOS

\begin{tabular}{|c|c|c|c|c|c|c|c|c|c|c|}
\hline & 2001 & 2002 & 2003 & 2004 & 2005 & 2006 & 2007 & 2008 & 2009 & 2010 \\
\hline REP & 24 & 24 & 23 & 24 & 25 & 23 & 24 & 26 & 26 & 25 \\
\hline T. $^{\text {a }}$ de la Ed. & 10 & 9 & 11 & 6 & 10 & 9 & 9 & 12 & 15 & 14 \\
\hline I\&A & 27 & 25 & 26 & 29 & 26 & 32 & 32 & 28 & 35 & 40 \\
\hline Psicodidáctica & 19 & 19 & 12 & 14 & 24 & 16 & 17 & 12 & 15 & 15 \\
\hline Comunicar & 46 & 56 & 52 & 50 & 59 & 64 & 47 & 44 & 43 & 41 \\
\hline Total & 126 & 133 & 124 & 123 & 144 & 144 & 129 & 122 & 134 & 135 \\
\hline
\end{tabular}




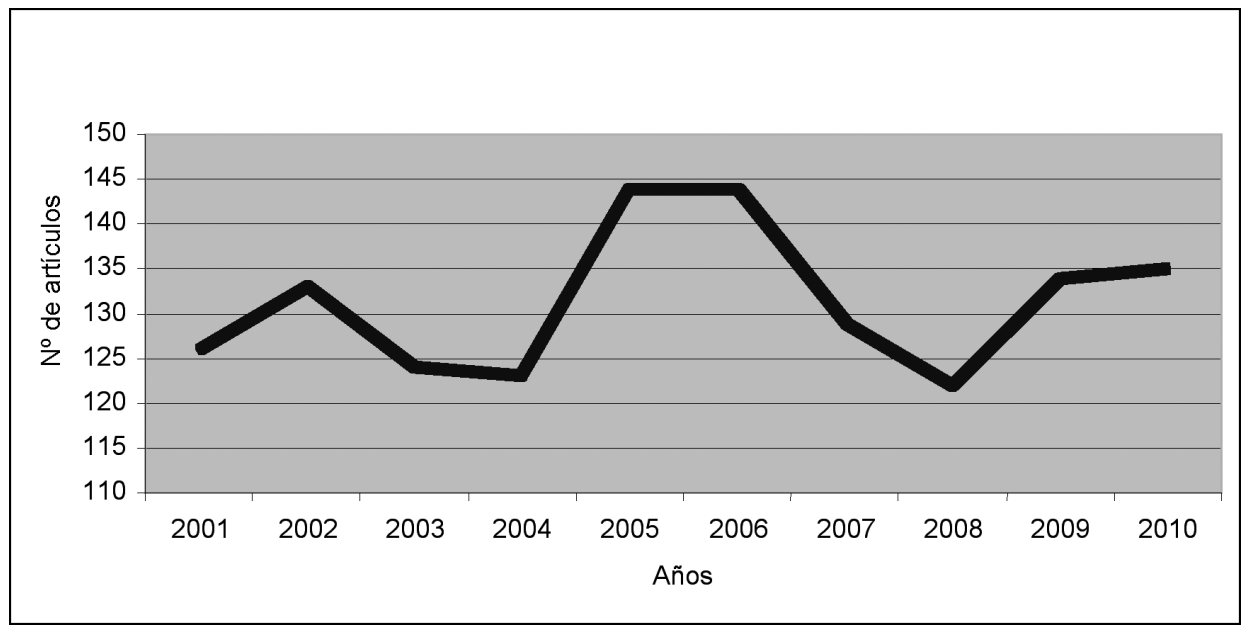

En general, no puede hablarse de un ascenso significativo en el número total de artículos ya que, por un lado, sólo existe una diferencia de +9 si se compara el primer año y el último y, por otro, la diferencia es únicamente de 22 artículos entre el año de menor producción científica, el 2008, y los de mayor producción científica, que son 2005 y 2006, lo cual hace que no se supere el 18\% de significatividad.

\section{GrÁFICO 2. EVOlUCión DEL NÚMERO DE ARTíCULOS PUBliCADOS POR REVISTA}

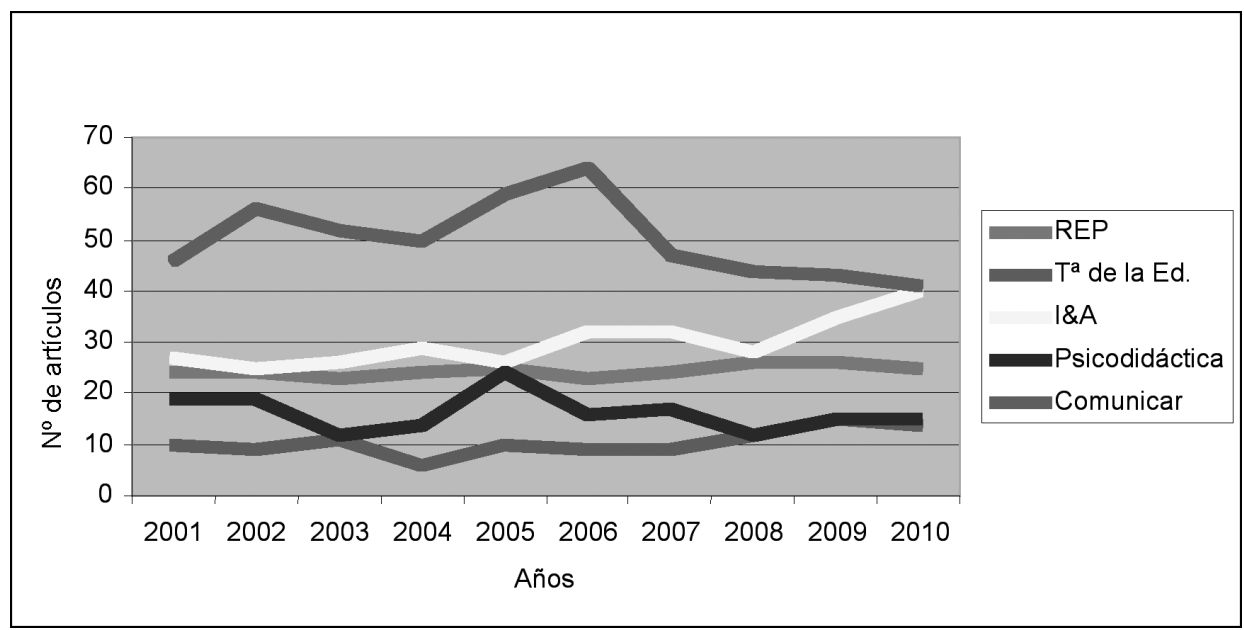


En el análisis de los datos de cada revista tampoco es posible apreciar ascensos elevados en ningún caso a excepción de Infancia y Aprendizaje, con una diferencia de 13 artículos entre el primer año y el último, y de 15 entre el año de menor y mayor producción. Asimismo, destaca el ligero repunte de Teoría de la Educación. Revista Interuniversitaria, que aumenta solo en 4 a pesar de duplicar de 1 número a 2 los volúmenes anuales, y el descenso en el número de artículos de Comunicar: Revista Científica de Comunicación y Educación y de Revista de Psicodidáctica, en las cuales se pueden observar algunas variaciones con picos en la primera entre los años 2006 y 2007, con 17 artículos de diferencia a la baja. Por su parte, la Revista Española de Pedagogía se muestra como la más regular en cuanto al número de publicaciones durante el periodo indicado.

Es interesante además prestar atención a la evolución que los distintos journals han experimentado desde su inclusión en el JCR. De esta forma, ninguna revista muestra diferencias significativas respecto a años anteriores, a excepción de Infancia y Aprendizaje que presenta un repunte de 12 artículos.

3.1.2.2. Artículos en secciones monográficas

TABla 3. ARTí́CUlOS EN SECCIONES MONOGRÁFICAS

\begin{tabular}{|c|c|c|c|c|c|}
\hline General & REP & T. ${ }^{a}$ de la Ed. & I\&A & Psicodidáctica & Comunicar \\
\hline 439 & 93 & 16 & 56 & 15 & 259 \\
\hline $34,41 \%$ & $38,11 \%$ & $15,24 \%$ & $18,66 \%$ & $9,20 \%$ & $51,59 \%$ \\
\hline
\end{tabular}

La Tabla 3 muestra diferencias importantes en el porcentaje de artículos que las diferentes revistas incluyen en secciones monográficas, destacando la revista Comunicar: Revista Científica de Comunicación y Educación, que dedica cada ejemplar a un tema monográfico, aunque incluye también otros artículos fuera de esta temática prefijada.

TABla 4. EVOLUCIÓN EN EL NÚMERO DE ARTÍCULOS EN SECCIONES MONOGRÁFICAS POR AÑOS

\begin{tabular}{|c|c|c|c|c|c|c|c|c|c|}
\hline 2001 & 2002 & 2003 & 2004 & 2005 & 2006 & 2007 & 2008 & 2009 & 2010 \\
\hline 38 & 31 & 51 & 41 & 65 & 36 & 46 & 58 & 39 & 33 \\
\hline
\end{tabular}

Si se atiende a la evolución por años no se percibe una tendencia clara al alza o a la baja, aunque sí algunos picos que podrían ser atribuidos a factores contingentes. 
3.1.3. Autoría de los trabajos

\subsubsection{Autores más prolíficos}

Se recogen a continuación los autores que destacan por su gran cantidad de producción científica:

TABla 5. AUTORES MÁS PROLÍFICOS

\begin{tabular}{|l|c|}
\hline \multicolumn{1}{|c|}{ Autores } & Número de artículos \\
\hline Medrano, Concepción & 11 \\
\hline Martín, Elena & 10 \\
\hline Cabero, Julio & 8 \\
\hline Rodrigo, María José & 8 \\
\hline Sánchez, Emilio & 8 \\
\hline Aierbe, Ana & 8 \\
\hline Montanero, Manuel & 8 \\
\hline Ortega Ruiz, Pedro & 8 \\
\hline Oliva, Alfredo & 8 \\
\hline
\end{tabular}

3.1.3.2. Autores más prolíficos por categorías del JCR

Si analizamos a los más prolíficos distinguiendo entre las categorías del JCR encontramos los siguientes datos:

TABla 6. Autores MÁS PROLÍFICOS EN EDUCACIÓN E INVESTIGACIÓN EDUCATIVA

\begin{tabular}{|l|c|}
\hline \multicolumn{1}{|c|}{ Autores } & Número de artículos \\
\hline Ortega Ruiz, Pedro & 8 \\
\hline Reyero García, David & 6 \\
\hline Gil Cantero, Fernando & 6 \\
\hline Peña Calvo, José Vicente & 5 \\
\hline Gargallo López, Bernardo & 5 \\
\hline García del Dujo, Ángel & 5 \\
\hline Bernal Guerrero, Antonio & 5 \\
\hline
\end{tabular}


JUAN LUIS FUENTES, DAVID LUQUE Y ERNESTO LÓPEZ GÓMEZ

ANÁLISIS BIBLIOMÉTRICO DE LAS REVISTAS ESPAÑOLAS DE EDUCACIÓN

TABla 7. Autores más Prolíficos en PSiCOLOGía DE LA EDUCACIÓN

\begin{tabular}{|l|c|}
\hline \multicolumn{1}{|c|}{ Autores } & Número de artículos \\
\hline Martín, Elena & 10 \\
\hline Oliva, Alfredo & 8 \\
\hline Rodrigo, María José & 8 \\
\hline Sánchez, Emilio & 8 \\
\hline Bausela, Esperanza & 7 \\
\hline López, Félix & 7 \\
\hline Del Barrio, Cristina & 7 \\
\hline Medrano, Concepción & 6 \\
\hline Vidal Abarca, Eduardo & 6 \\
\hline Jiménez, Juan & 6 \\
\hline Madariaga, José & 5 \\
\hline Etxebarría, Itziar & 5 \\
\hline Fuentes, María Jesús & 5 \\
\hline
\end{tabular}

\subsubsection{Colaboración entre autores}

TABLA 8. COLABORACiÓN ENTRE AUTORES

\begin{tabular}{|c|c|c|c|c|c|c|}
\hline & General & REP & T. ${ }^{\text {a }}$ de la Ed. & I\&A & Psicodidáctica & Comunicar \\
\hline $\begin{array}{c}\bar{X} \text { Autores } \\
\text { por artículo }\end{array}$ & 1,82 & 1,81 & 1,54 & 2,62 & 2,06 & 1,32 \\
\hline $\begin{array}{l}\text { Artículos } \\
\text { colectivos }\end{array}$ & $\begin{array}{c}581 \\
44,21 \%\end{array}$ & $\begin{array}{c}97 \\
39,75 \%\end{array}$ & $\begin{array}{c}41 \\
39,05 \%\end{array}$ & $\begin{array}{c}236 \\
78,66 \%\end{array}$ & $\begin{array}{c}83 \\
50,92 \%\end{array}$ & $\begin{array}{c}124 \\
24,70 \%\end{array}$ \\
\hline $\begin{array}{c}\bar{X} \text { Autores } \\
\text { en artículos } \\
\text { colectivos }\end{array}$ & 2,84 & 3,04 & 2,39 & 3,06 & 3,07 & 2,31 \\
\hline
\end{tabular}

El grado de colaboración entre autores muestra uno de los ejes que en mayor medida viene siendo desarrollado en el nuevo Espacio Europeo de Educación Superior: el trabajo en equipo (Apodaca, 2006; León del Barco y Latas, 2007). Para abordar esta cuestión hemos identificado el habitual grado de coautoría (Russell, Madera-Jaramillo y Ainsworth, 2009), esto es, número de autores por artículo, pero también el porcentaje de artículos individuales o colectivos que se publican en las diferentes revistas.

Así pues, encontramos que Infancia y Aprendizaje alcanza un alto grado de coautoría cercano a 3 autores por artículo, seguida a bastante distancia de Revista de Psicodidáctica. En el otro extremo se encuentran Teoría de la Educación. Revista Interuniversitaria y, muy destacadamente, Comunicar: Revista Científica de Comunicación y Educación. Con respecto al número de artículos colectivos encontramos datos muy similares. Sin embargo, esta variable nos muestra también 
que los artículos individuales son mayoría en 3 de los 5 journals, aquellos que mostraban datos más bajos en la variable anterior.

Por último, recogemos el número de autores que participan en los artículos colectivos de tal forma que puede conocerse la media de autores que suelen conformar las investigaciones grupales. Los resultados generales muestran que los investigadores colaboran en grupos formados por cerca de 3 personas, mientras que los datos por revistas indican que hay dos categorías de revistas claramente diferenciadas. Por un lado, Revista Española de Pedagogí, Infancia y Aprendizaje y Revista de Psicodidáctica con grupos de investigadores con más de 3 miembros. Por otro, Teoría de la Educación. Revista Interuniversitaria y Comunicar: Revista Científica de Comunicación y Educación, donde el número de autores en artículos colectivos se sitúa más cercano a 2 investigadores.

Unido a esto, puede resultar de interés observar la evolución de estos datos durante el periodo de tiempo estudiado.

TABLA 9. EVOLUCIÓN EN LA COLABORACiÓN ENTRE AUTORES

\begin{tabular}{|c|c|c|c|c|c|c|c|c|c|c|}
\hline & 2001 & 2002 & 2003 & 2004 & 2005 & 2006 & 2007 & 2008 & 2009 & 2010 \\
\hline $\begin{array}{c}\bar{X} \text { Autores } \\
\text { por artículo }\end{array}$ & 1,67 & 1,70 & 1,77 & 1,72 & 1,46 & 1,55 & 1,72 & 2,08 & 2,20 & 2,35 \\
\hline $\begin{array}{c}\text { Artículos } \\
\text { colectivos }\end{array}$ & $47,30 \%$ & $\begin{array}{c}53 \\
40,15 \%\end{array}$ & $\begin{array}{c}48,71 \% \\
48\end{array}$ & $\begin{array}{c}51 \\
41,46 \%\end{array}$ & $\begin{array}{c}46 \\
32,17 \%\end{array}$ & $\begin{array}{c}54 \\
37,76 \%\end{array}$ & $\begin{array}{c}52 \\
40,31 \%\end{array}$ & $\begin{array}{c}63 \\
51,64 \%\end{array}$ & $\begin{array}{c}78 \\
59,54 \%\end{array}$ & $\begin{array}{c}89 \\
69,93 \%\end{array}$ \\
\hline
\end{tabular}

GRÁFICO 3. EVOLUCiÓN EN LA COLABORACIÓN ENTRE AUTORES

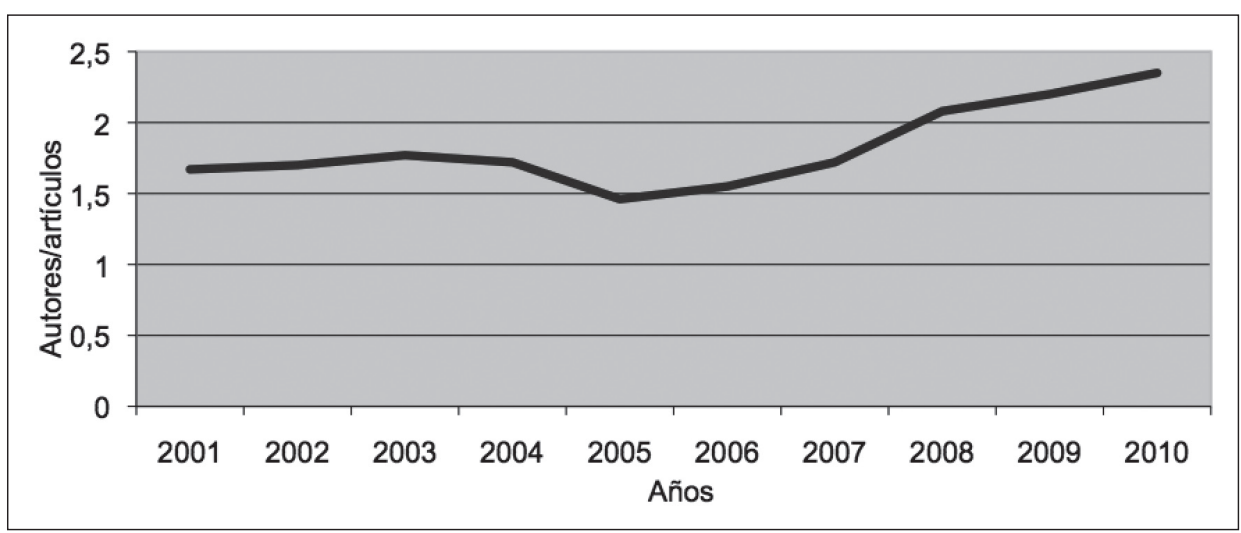

Así pues, se aprecia una subida de 0,68 autores por artículo que alcanza el grado máximo en el último año analizado. 


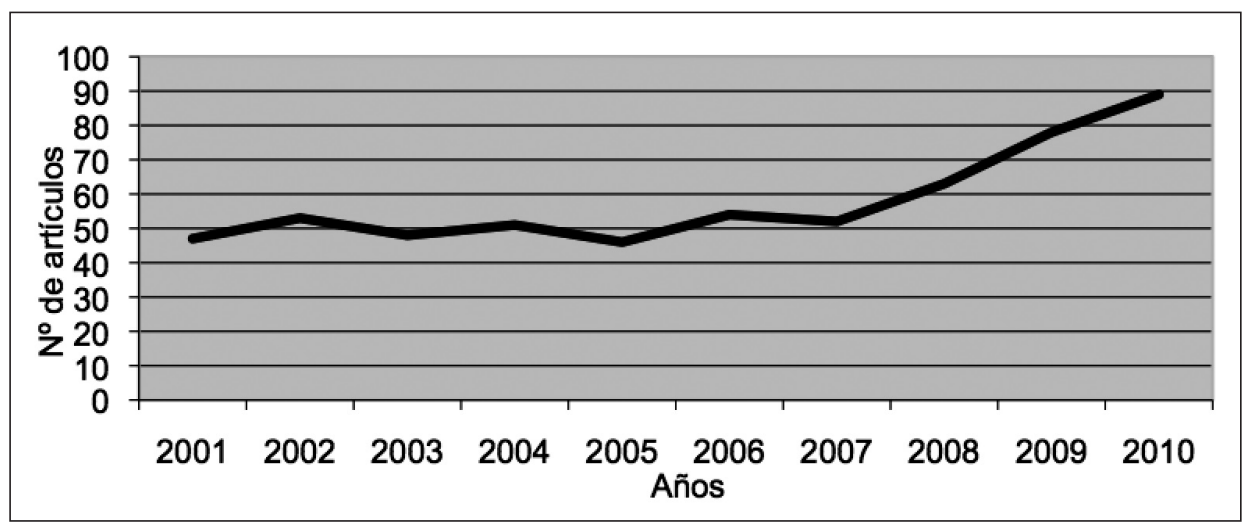

Atendiendo a la evolución del número de artículos colectivos nos encontramos con un ascenso muy significativo en el periodo de tiempo analizado, en el que casi se duplica su presencia en el total de investigaciones publicadas.

\subsubsection{Sexo de los autores}

TABla 10. SEXO De LOS AUTORES

\begin{tabular}{|c|c|c|c|c|c|c|}
\hline & General & REP & T. ${ }^{\text {a }}$ de la Ed. & I\&A & Psicodidáctica & Comunicar \\
\hline Hombres & $52,2 \%$ & $58,14 \%$ & $67,28 \%$ & $40,91 \%$ & $49,68 \%$ & $59,06 \%$ \\
\hline Mujeres & $47,8 \%$ & $41,86 \%$ & $32,72 \%$ & $59,09 \%$ & $50,32 \%$ & $40,94 \%$ \\
\hline
\end{tabular}

Aunque los datos generales muestran cierta igualdad entre hombres y mujeres, en casi todas las revistas excepto en Revista de Psicodidáctica hay diferencias destacables por encima del $10 \%$ en un sentido u otro. Así pues, en Infancia y Aprendizaje el porcentaje de mujeres es superior al de los hombres llegando a alcanzar casi 20 puntos de diferencia. Sin embargo, en Comunicar: Revista Científica de Comunicación y Educación y en la Revista Española de Pedagogía el 20\% es superior en los articles firmados por hombres, mientras que en Teoría de la Educación. Revista Interuniversitaria la diferencia está cerca del 40\%.

Si analizamos las revistas dependiendo de la categoría del JCR no parece haber una tendencia clara por la que hombres o mujeres opten por un ámbito $\mathrm{u}$ otro en sus publicaciones. Pues aunque en las dos revistas de la categoría "Educación e Investigación Educativa" muestran mayor presencia masculina, la categoría de "Psicología de la Educación" muestra datos diferentes. En Revista de Psicodidáctica no hay diferencias mientras que por el contrario en Infancia $y$ Aprendizaje aparecen. 
JUAN LUIS FUENTES, DAVID LUQUE Y ERNESTO LÓPEZ GÓMEZ ANÁLISIS BIBLIOMÉTRICO DE LAS REVISTAS ESPAÑOLAS DE EDUCACIÓN

3.1.4. Instituciones representadas

3.1.4.1. Instituciones de origen de los autores

TABLA 11. INSTITUCIONES DE ORIGEN DE LOS AUTORES

\begin{tabular}{|c|c|c|c|c|c|c|}
\hline & General & REP & T. a de la Ed. & I\&A & Psicodidáctica & Comunicar \\
\hline $\begin{array}{c}\text { Universidades } \\
\text { Españolas }\end{array}$ & $76,9 \%$ & $\begin{array}{c}394 \\
89,14 \% \\
\end{array}$ & $\begin{array}{c}150 \\
93,17 \% \\
\end{array}$ & $\begin{array}{c}642 \\
81,68 \%\end{array}$ & $\begin{array}{c}281 \\
87,81 \%\end{array}$ & $\begin{array}{c}359 \\
53,98 \%\end{array}$ \\
\hline $\begin{array}{l}\text { Universidades } \\
\text { Extranjeras }\end{array}$ & $13,8 \%$ & $\begin{array}{c}25 \\
5,66 \%\end{array}$ & $\begin{array}{c}9 \\
5,59 \%\end{array}$ & $\begin{array}{c}95 \\
12,09 \% \\
\end{array}$ & $\begin{array}{c}25 \\
7,81 \%\end{array}$ & $\begin{array}{c}173 \\
26,06 \%\end{array}$ \\
\hline $\begin{array}{l}\text { Centros de } \\
\text { Educación } \\
\text { no superior }\end{array}$ & $2,9 \%$ & $\begin{array}{c}4 \\
0,90 \%\end{array}$ & $\begin{array}{c}0 \\
0 \%\end{array}$ & $\begin{array}{c}10 \\
1,27 \%\end{array}$ & $\begin{array}{c}3 \\
0,94 \%\end{array}$ & $\begin{array}{c}51 \\
7,67 \%\end{array}$ \\
\hline $\begin{array}{c}\text { Otras } \\
\text { instituciones }\end{array}$ & $6,4 \%$ & $\begin{array}{c}19 \\
4,30 \%\end{array}$ & $\begin{array}{c}2 \\
1,24 \%\end{array}$ & $\begin{array}{c}39 \\
4,96 \%\end{array}$ & $\begin{array}{c}11 \\
3,44 \%\end{array}$ & $\begin{array}{c}82 \\
12,33 \%\end{array}$ \\
\hline
\end{tabular}

GrÁfico 5. InStituciones DE ORIGEN DE LOS AUTORES

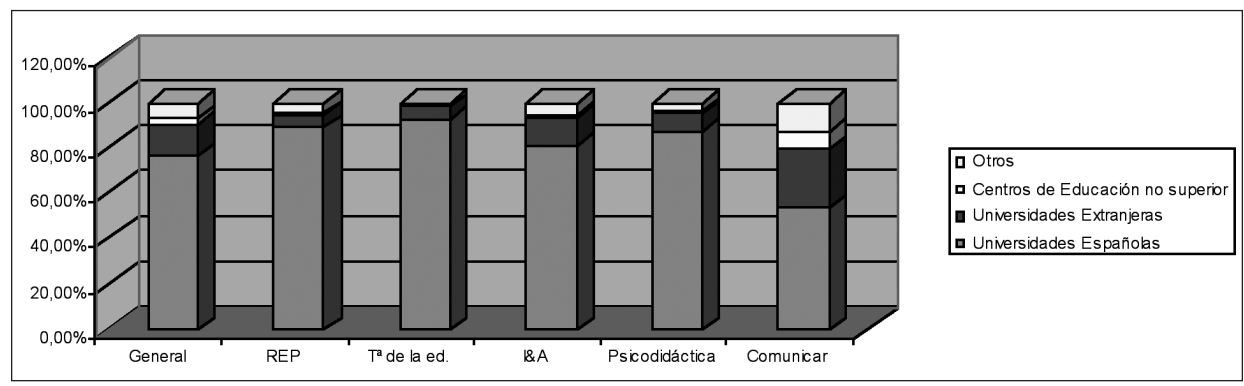

Como se puede observar (Tabla 10) en la variable "Universidades Españolas" se obtienen unos porcentajes similares en todas las revistas excepto en Comunicar: Revista Científica de Comunicación y Educación donde el porcentaje desciende considerablemente. Esta tendencia se mantiene en cierta medida en la variable "Universidades extranjeras" donde a excepción de esta revista, que publica a un número sensiblemente mayor de autores procedentes de universidades extranjeras, todas mantienen números relativamente bajos respecto del total de artículos publicados. Comunicar: Revista Científica de Comunicación y Educación, por tanto, se muestra como la publicación más heterogénea en este sentido pues además de las dos variables anteriores mantiene también la tendencia en el resto. Cabe destacar también que el origen universitario de los autores, ya sea español o extranjero, supone en las otras 4 revistas alrededor del 95\% de las publicaciones, mientras que en la revista del "Grupo Comunicar", aunque sigue siendo mayoritario, se queda en el $80 \%$. 
Resulta interesante detenerse en los centros de educación no superior que cuentan con mayor representación en "Comunicar", donde han publicado autores de estas instituciones un total de 51 artículos. Si acaso le sigue Infancia y Aprendizaje, pero muy de lejos, con sólo 10 artículos. En el resto su presencia no es significativa. En conjunto publican 53 artículos procedentes de autores de centros de educación no superior, de los cuales el 39,62\% son Institutos de Educación Secundaria y el 30,19\% escuelas de infantil y primaria.

\subsubsection{Instituciones más prolíficas}

Dentro del ámbito de las instituciones más prolíficas se observa que se han publicado artículos cuya procedencia alcanza un total de 414 centros diferentes. Las instituciones con mayor presencia son las siguientes ${ }^{4}$ :

\section{TABLA 12. INSTITUCIONES CON MAYOR PRESENCIA}

\begin{tabular}{|l|c|c|}
\hline \multicolumn{1}{|c|}{ Instituciones } & \multicolumn{2}{c|}{ Participación en artículos (\%) } \\
\hline Universidad del País Vasco & 236 & 9,87 \\
\hline Universidad de Sevilla & 135 & 5,64 \\
\hline Universidad de Barcelona & 92 & 3,84 \\
\hline Universidad de Málaga & 88 & 3,68 \\
\hline Universidad Autónoma de Madrid & 86 & 3,59 \\
\hline Universidad de Valencia & 85 & 3,55 \\
\hline Universidad Complutense de Madrid & 84 & 3,51 \\
\hline Universidad de Granada & 84 & 3,51 \\
\hline Universidad de Salamanca & 84 & 3,51 \\
\hline Universidad de La Laguna & 83 & 3,47 \\
\hline
\end{tabular}

\subsubsection{Instituciones de educación no superior más prolíficas}

En relación a los centros de educación no superior más prolíficos destacan, por su representatividad, el "IES Pablo Neruda", el "IES V Centenario" y el "IES San Sebastián" con 5, 5 y 3 artículos respectivamente.

4. La tabla que se presenta no indica qué institución publica mayor número de artículos, sino que hay un número mayor de autores que ejercen su labor en la institución correspondiente que ha participado en artículos publicados, pero no especifica que los artículos sean colectivos o individuales. 


\subsection{Elementos controvertidos}

\subsubsection{Endogamia}

La idea de endogamia, cuyo uso se ha extendido en diversos ámbitos como el político pero entre los que se encuentra por supuesto el universitario, es un concepto que no cuenta sin embargo con una definición cristalina en la literatura científica. A efectos de la presente investigación entendemos por endogamia la tendencia de los autores a publicar los resultados de sus investigaciones en revistas con las que mantienen una estrecha relación, sea porque forman parte del organigrama científico o porque son las editadas por la universidad en la que trabajan. Ahora bien, ¿a partir de qué punto podemos hablar de un nivel endogámico alto? La "Fundación Española para la Ciencia y la Tecnología" $(2007,75)$ propone dos criterios de calidad científica que nos permiten determinar un límite a partir del cual las prácticas endogámicas comienzan a ser perniciosas para la difusión del conocimiento:

- Más del 80\% de los autores serán externos al Comité Editorial.

- Más del 80\% de los autores serán externos a la organización editora de la revista.

Así pues, con las premisas expuestas vamos a analizar aquí la endogamia en las revistas de educación aceptadas en el $J C R$ durante la primera década del siglo XXI, en función de los dos criterios ya adelantados:

- Endogamia editorial. Artículos publicados por autores que forman parte del organigrama científico de la revista.

- Endogamia universitaria. Publicaciones en las que participan autores que forman parte de la universidad que edita la revista.

\subsubsection{Endogamia editorial}

Si miramos a los consejos editoriales de las cinco revistas españolas incluidas en el JCR se observa que no existen criterios comunes en la estructura de los organigramas. La primera diferencia reside en el número de miembros que forman parte de los diferentes órganos, donde hay variaciones importantes en número desde los 25 que conforman la Revista Española de Pedagogía, los 60 de Teoría de la Educación. Revista Interuniversitaria, los 40 de Revista de Psicodidáctica, los 77 de Infancia y Aprendizaje y los 82 de Comunicar. Por otro lado, están formados por diferentes órganos tal y como se muestra en la siguiente tabla. 
TABla 13. ORganigRAmas DE LAS REVISTAS

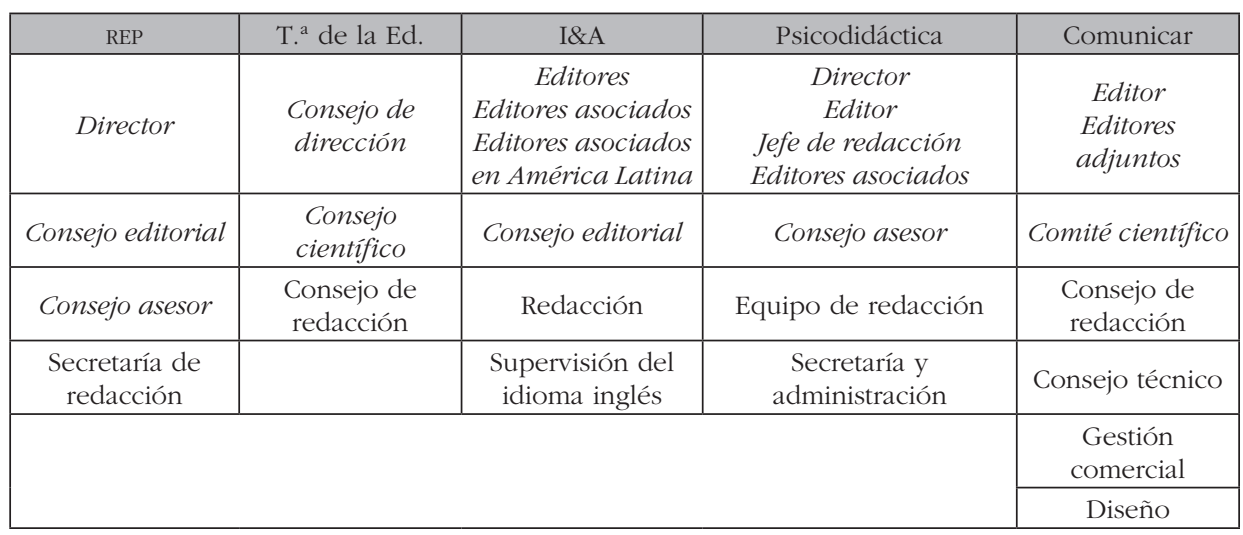

Para poder comparar con criterios similares hemos seleccionado los órganos de carácter científico, excluyendo así las secretarías, equipos y consejos de redacción y los órganos administrativos, comerciales y de diseño. Consideraremos de esta forma endogamia editorial cuando publique en la propia revista algún miembro de los órganos en cursiva del cuadro anterior, que llamaremos organigrama científico de las revistas y que en número son los que se muestran en la Tabla 14.

TABla 14. NÚMERO DE LOS COMPONENTES DE LOS ORGANIGRAMAS CIENTÍFICOS

\begin{tabular}{|c|c|c|c|c|}
\hline REP & T. ${ }^{\text {a de la Ed. }}$ & I\&A & Psicodidáctica & Comunicar \\
\hline 20 & 47 & 76 & 39 & 35 \\
\hline
\end{tabular}

Por último, es necesario señalar que en sentido estricto para poder determinar si las publicaciones son realizadas por miembros del organigrama científico de la revista se necesitaría acudir al momento concreto de la publicación del artículo o incluso al momento en que éste es aceptado para publicarse, lo que no siempre coincide con su efectiva publicación. La dificultad y laboriosidad para acceder a estos datos nos ha llevado a elegir otro criterio que aun no siendo tan ajustado nos posibilita un conocimiento aceptable de este grado de endogamia. El criterio a seguir ha sido el de determinar si es parte de alguno de los órganos de la revista descritos en el momento actual. Puesto que no analizamos un periodo de tiempo muy amplio ni parece haber muchas variaciones en la composición de los organigramas de las revistas, este criterio es válido para nuestro propósito. 
GRÁFICO 6. PORCENTAJE DE AUTORES QUE SON MIEMBROS DEL ORGANIGRAMA CIENTÍFICO DE LA REVISTA EN LA QUE PUBLICAN

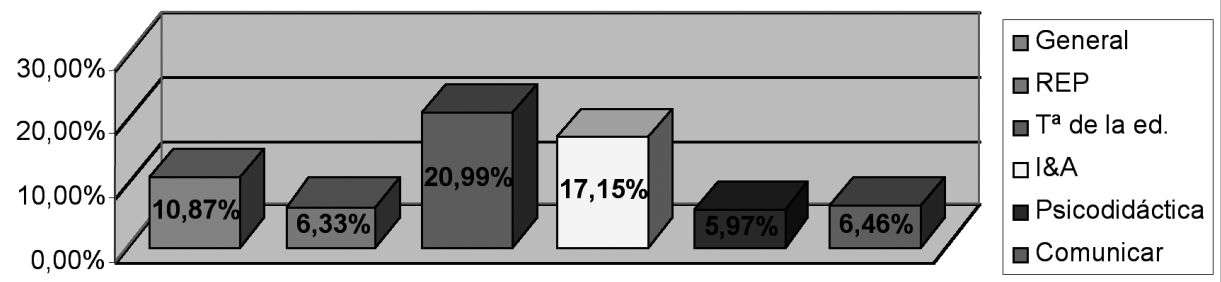

Mientras que como podemos observar la Revista Española de Pedagogía -6,33\%, 28 artículos de 442-, Revista de Psicodidáctica -5,97\%, 20 de 335- y Comunicar: Revista Científica de Comunicación y Educación -6,46\%, 43 de 665- se mueven en porcentajes similares, Infancia y Aprendizaje -17,15\%, 135 de 787- y Teoría de la Educación. Revista Interuniversitaria-20,99\%, 34 de 162muestran datos superiores. Coincide esto con que también se han considerado números más amplios de miembros del Consejo en estas 2 revistas que en el resto, pero la composición de los organigramas es una decisión que compete a las mismas revistas.

A continuación parece interesante analizar si los autores más prolíficos de cada revista pertenecen a sus respectivos organigramas científicos:

Tabla 15. Autores más prolíficos de la ReVISTA Española de Pedagogía

\begin{tabular}{|l|c|c|}
\hline \multicolumn{1}{|c|}{ Autores } & $\begin{array}{c}\text { Número } \\
\text { de artículos }\end{array}$ & $\begin{array}{c}\text { Miembro del } \\
\text { organigrama científico }\end{array}$ \\
\hline Montanero, Manuel & 4 & NO \\
\hline Gargallo López, Bernardo & 3 & NO \\
\hline Ortega Ruiz, Pedro & 3 & NO \\
\hline Tejedor Tejedor, Francisco Javier & 3 & SÍ \\
\hline Quintana Cabanas, José María & 3 & NO \\
\hline Gobernado Arribas, Rafael & 3 & NO \\
\hline Reyero García, David & 3 & SÍ \\
\hline Ibáñez-Martín, José Antonio & 3 & \\
\hline
\end{tabular}


TABla 16. Autores más PROLÍFICOS DE TEORÍA DE LA EDUCACIÓN. REVISTA INTERUNIVERSITARIA

\begin{tabular}{|l|c|c|}
\hline \multicolumn{1}{|c|}{ Autores } & $\begin{array}{c}\text { Número de } \\
\text { artículos }\end{array}$ & $\begin{array}{c}\text { Miembros del } \\
\text { organigrama científico }\end{array}$ \\
\hline Ortega Ruiz, Pedro & 5 & SÍ \\
\hline Gil Cantero, Fernando & 4 & SÍ \\
\hline García del Dujo, Ángel & 4 & NO \\
\hline Rodríguez Menéndez, María del Carmen & 3 & NO \\
\hline Reyero García, David & 3 & Sí \\
\hline Touriñán, José Manuel & 3 & SÍ \\
\hline Jover, Gonzalo & 3 & NO \\
\hline Martín García, Antonio & 3 & NO \\
\hline Bernal Guerrero, Antonio & 3 & SÍ \\
\hline Muñoz Rodríguez, José Manuel & 3 & SÍ \\
\hline Escámez Sánchez, Juan & 3 & NO \\
\hline Mínguez Vallejos, Ramón & 3 & SÍ \\
\hline Peña Calvo, José Vicente & 3 & \\
\hline
\end{tabular}

\section{TABla 17. Autores más PROLÍFICOS DE INFANCIA Y APRENDIZAJE}

\begin{tabular}{|c|c|c|}
\hline Autores & $\begin{array}{l}\text { Número de } \\
\text { artículos }\end{array}$ & $\begin{array}{c}\text { Miembros del } \\
\text { organigrama científico }\end{array}$ \\
\hline Martín, Elena & 10 & Sí \\
\hline Sánchez, Emilio & 8 & Sí \\
\hline Rodrigo, María José & 8 & SÍ \\
\hline Oliva, Alfredo & 8 & SÍ \\
\hline López, Félix & 7 & SÍ \\
\hline Vidal Abarca, Eduardo & 6 & Sí \\
\hline Jiménez, Juan & 6 & $\mathrm{NO}$ \\
\hline Del Barrio, Cristina & 6 & $\mathrm{NO}$ \\
\hline Fuentes, María Jesús & 5 & $\mathrm{NO}$ \\
\hline Mauri, Teresa & 4 & Sí \\
\hline \begin{tabular}{|l|} 
Del Río, Pablo \\
\end{tabular} & 4 & $\mathrm{NO}$ \\
\hline Garaigordobil, Maite & 4 & $\mathrm{NO}$ \\
\hline Peralta, Olga & 4 & $\mathrm{NO}$ \\
\hline Etxeberría, Itziar & 4 & Sí \\
\hline Onrubia, Javier & 4 & $\mathrm{NO}$ \\
\hline \begin{tabular}{|l|} 
Serrat, Elisabet \\
\end{tabular} & 4 & SÍ \\
\hline Sarría, Encarnación & 4 & Sí \\
\hline González, Antonia & 4 & $\mathrm{NO}$ \\
\hline Parra, Águeda & 4 & $\mathrm{NO}$ \\
\hline Pozo, Juan Ignacio & 4 & SÍ \\
\hline Cantero, María José & 4 & $\mathrm{NO}$ \\
\hline
\end{tabular}


TABla 18. Autores más PROlíficos de REVISTA DE PSICODIDÁCTICA

\begin{tabular}{|l|c|c|}
\hline \multicolumn{1}{|c|}{ Autores } & N. ${ }^{\circ}$ de artículos & $\begin{array}{c}\text { Miembros del } \\
\text { organigrama científico }\end{array}$ \\
\hline Bausela, Esperanza & 7 & NO \\
\hline Madariaga, José & 5 & NO \\
\hline Medrano, Concepción & 5 & NO \\
\hline Carbonero, Miguel Ángel & 4 & NO \\
\hline Merino, Enrique & 4 & NO \\
\hline Lobato, Clemente & 4 & NO \\
\hline Goñi, Alfredo & 4 & Sí \\
\hline Maia, Julián & 4 & NO \\
\hline Aierbe, Ana & 3 & NO \\
\hline Palacios, Santiago & 3 & NO \\
\hline Núñez, José & 3 & NO \\
\hline Uriarte, Juan de Dios & 3 & NO \\
\hline Goikoetxea, Javier & 3 & NO \\
\hline Latorre, María José & 3 & NO \\
\hline Ruiz de Azúa, Sonia & 3 & NO \\
\hline
\end{tabular}

TABla 19. AutORES MÁS PROLÍFICOS DE

COMUNICAR: REVISTA CIENTÍFICA DE COMUNICACIÓN Y EDUCACIÓN

\begin{tabular}{|l|c|c|}
\hline \multicolumn{1}{|c|}{ Autores } & N. ${ }^{\circ}$ de artículos & $\begin{array}{c}\text { Miembros del } \\
\text { organigrama científico }\end{array}$ \\
\hline Cabero, Julio & 8 & NO \\
\hline Martínez Salanova, Enrique & 7 & Sí \\
\hline Aguaded, José Ignacio & 5 & Sí \\
\hline Marín Díaz, Verónica & 4 & NO \\
\hline Santibáñez, Josefina & 4 & NO \\
\hline Pedroso, Tomás & 4 & NO \\
\hline Gabelas, José Antonio & 3 & NO \\
\hline Contín, Silvia Andrea & 3 & NO \\
\hline Martínez Fresneda, Humberto & 3 & NO \\
\hline Rodríguez Fuentes, Antonio & 3 & NO \\
\hline Clarembeaux, Michel & 3 & SÍ \\
\hline Pérez Tornero, José Manuel & 3 & NO \\
\hline Ricoy, María del Carmen & 3 & NO \\
\hline Pindado, Julián & 3 & NO \\
\hline Aznar, Inmaculada & 3 & NO \\
\hline
\end{tabular}



ANÁLISIS BIBLIOMÉTRICO DE LAS REVISTAS ESPAÑOLAS DE EDUCACIÓN

\begin{tabular}{|l|c|c|}
\hline \multicolumn{1}{|c|}{ Autores } & N. ${ }^{\circ}$ de artículos & $\begin{array}{c}\text { Miembros del } \\
\text { organigrama científico }\end{array}$ \\
\hline García Matilla, Agustín & 3 & NO \\
\hline Jaramillo, Alejandro & 3 & NO \\
\hline Sedeño, Ana María & 3 & NO \\
\hline Fontcuberta, Mar & 3 & SÍ \\
\hline Reia Baptista, Vitor & 3 & NO \\
\hline De Aguilera, Miguel & 3 & NO \\
\hline Millán Paredes, Tatiana & 3 & NO \\
\hline Méndez Garrido, Juan Manuel & 3 & NO \\
\hline Hinojo, Francisco Javier & 3 & \\
\hline
\end{tabular}

\subsubsection{Endogamia universitaria}

Se analiza ahora el grado de endogamia dependiendo del porcentaje de autores que publican en las revistas y pertenecen a la universidad donde éstas se editan. Este análisis es posible realizarlo tan sólo con las revistas Teoría de la Educación. Revista Interuniversitaria, que edita la Universidad de Salamanca, y Revista de Psicodidáctica, por la Universidad del País Vasco.

TABLA 20. INSTITUCIONES CON MAYOR PRESENCIA EN TEORÍA DE LA EDUCACIÓN. REVISTA INTERUNIVERSITARIA

\begin{tabular}{|l|c|}
\hline \multicolumn{1}{|c|}{ Instituciones } & $\begin{array}{c}\text { Participación } \\
\text { en artículos }\end{array}$ \\
\hline Universidad de Valencia & $14,2 \%$ \\
\hline Universidad Complutense de Madrid & $14,2 \%$ \\
\hline Universidad de Salamanca & $9,3 \%$ \\
\hline Universidad de Murcia & $8,02 \%$ \\
\hline Universidad de Sevilla & $4,94 \%$ \\
\hline Universidad de Barcelona & $4,94 \%$ \\
\hline Universidad de Santiago de Compostela & $4,94 \%$ \\
\hline Universidad de Oviedo & $4,94 \%$ \\
\hline Universidad Nacional de Educación a Distancia & $4,32 \%$ \\
\hline Universidad Autónoma de Barcelona & $3,7 \%$ \\
\hline
\end{tabular}

En la primera se observa que las instituciones con mayor presencia en la procedencia de los autores son la Universidad de Valencia y la Complutense de Madrid seguidas por la propia Universidad de Salamanca. 
JUAN LUIS FUENTES, DAVID LUQUE Y ERNESTO LÓPEZ GÓMEZ ANÁLISIS BIBLIOMÉTRICO DE LAS REVISTAS ESPAÑOLAS DE EDUCACIÓN

TABla 21. INSTITUCIONES CON MAYOR PRESENCIA EN REVISTA DE PSICODIDÁCTICA

\begin{tabular}{|l|c|}
\hline \multicolumn{1}{|c|}{ Instituciones } & \multicolumn{1}{|c|}{$\begin{array}{c}\text { Participación } \\
\text { en artículos }\end{array}$} \\
\hline Universidad del País Vasco & $45,55 \%$ \\
\hline Universidad de Oviedo & $4,5 \%$ \\
\hline Universidad de Valladolid & $3,6 \%$ \\
\hline Universidad de Lérida & $2,7 \%$ \\
\hline Universidad de Granada & $2,4 \%$ \\
\hline Universidad de León & $2,1 \%$ \\
\hline Universidad de A Coruña & $2,1 \%$ \\
\hline Universidad de Barcelona & $1,8 \%$ \\
\hline Universidad Autónoma de Madrid & $1,5 \%$ \\
\hline
\end{tabular}

Mientras que en la Revista de Psicodidáctica casi la mitad de sus artículos son publicados por autores de la universidad donde se edita. Muy de lejos se sitúan la Universidad de Oviedo y la de Valladolid.

3.2.2. Investigaciones financiadas

TABla 22. TRABAjOS FinANCIADOS*

\begin{tabular}{|c|c|c|c|c|c|}
\hline General & REP & T. $^{\text {a }}$ de la Ed. & I\&A & Psicodidáctica & Comunicar \\
\hline 196 & 8 & 5 & 137 & 13 & 33 \\
$14,91 \%$ & $3,28 \%$ & $4,76 \%$ & $45,66 \%$ & $7,98 \%$ & $6,57 \%$ \\
\hline
\end{tabular}

* Trabajos que especifican que han recibido ayudas a la investigación que motiva la publicación.

A la luz de los resultados anteriores se observa escasa financiación en casi todas las revistas excepto en Infancia y Aprendizaje donde cerca de la mitad de los artículos publicados han recibido apoyo económico. Asimismo, podemos decir que el 69,89\% de los artículos financiados del total de las 5 revistas están en esta revista.

Por otro lado, puede señalarse que los trabajos publicados en las revistas del ámbito de "Psicología de la Educación" reciben mayor financiación que los del resto de ámbitos.

TABla 23. Evolución de los aRTículos FinANCiAdos POR AÑOS

\begin{tabular}{|c|c|c|c|c|c|c|c|c|c|}
\hline 2001 & 2002 & 2003 & 2004 & 2005 & 2006 & 2007 & 2008 & 2009 & 2010 \\
\hline 15 & 17 & 10 & 9 & 18 & 13 & 21 & 22 & 32 & 37 \\
\hline $11,9 \%$ & $12,78 \%$ & $8,06 \%$ & $7,32 \%$ & $12,5 \%$ & $9,03 \%$ & $16,28 \%$ & $18,03 \%$ & $23,88 \%$ & $27,41 \%$ \\
\hline
\end{tabular}


GRÁFICO 7. EVOLUCIÓN EN EL NÚMERO DE ARTíCUlOS FINANCIADOS

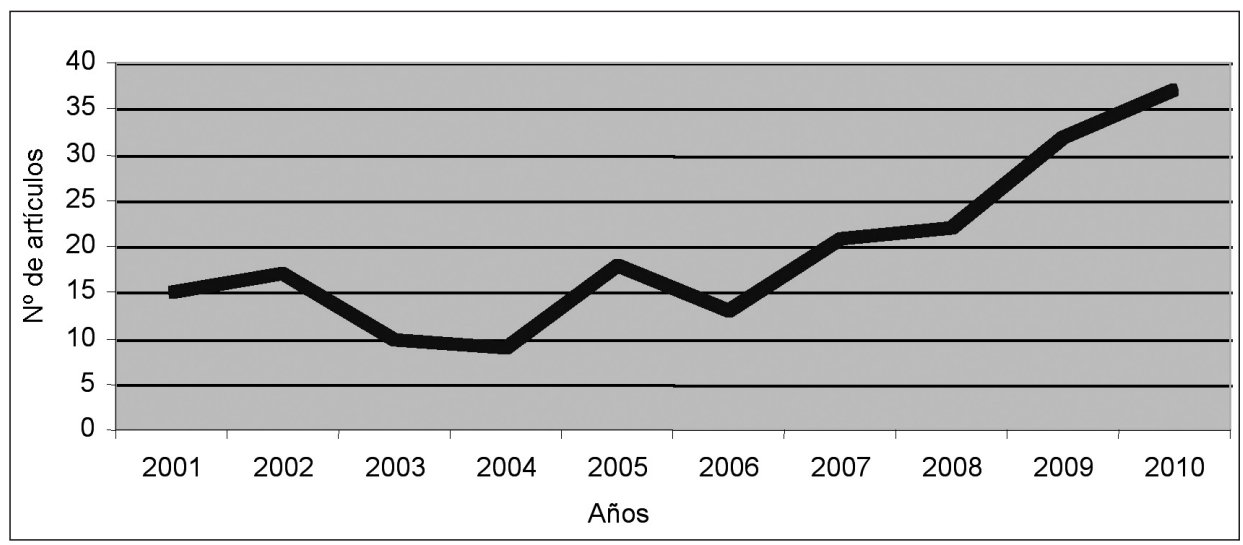

Con respecto a la evolución del número de artículos financiados, se observa una tendencia al alza especialmente a partir de 2006, lo que coincide con el momento en que las revistas empiezan a aparecer en el JCR. El número de articles más bajo se encuentra en 2004, con sólo 9, y el más alto con 37 en 2010, esto es, una diferencia de 28 artículos financiados más y una diferencia de 20 puntos porcentuales.

Otro aspecto interesante a analizar se deriva de la relación entre el trabajo colaborativo y la financiación. De esta manera encontramos que de los 584 artículos colectivos el $25,51 \%$ reciben financiación, mientras que si atendemos a las investigaciones individuales, el porcentaje baja hasta el 6,57\%.

\subsubsection{Internacionalización}

Relacionado con el grado de endogamia se encuentra este último criterio dirigido a evaluar el grado en que las revistas españolas de educación incluidas en el $J C R$ constituyen una referencia para difundir las producciones académicas de autores originarios de países diferentes al nuestro. Se analizará esto a través de los siguientes aspectos.

En primer lugar, se observará el porcentaje de autores de universidades extranjeras que han publicado en los últimos 10 años en las revistas objeto de nuestro estudio. Asimismo, se analizarán las instituciones y autores más productivos fuera de las fronteras españolas, desglosando los datos por revistas y áreas, considerando también la evolución en este periodo de tiempo que termina con la finalización del plazo para la implantación en las universidades españolas del Espacio Europeo de Educación Superior (EEEs) que, como es sabido, sitúa entre sus objetivos la internacionalización de la educación superior.

En segundo lugar, se estudiará el porcentaje de artículos publicados en una lengua diferente al castellano. Y, por último, el número de traducciones que pueden encontrarse. 
3.2.3.1. Autores e instituciones extranjeras en las revistas españolas de educación del JCR

TABla 24. Porcentaje DE AUTORES PROCEDENTES DE UNIVERSIDADES EXTRANJERAS

\begin{tabular}{|c|c|c|c|c|c|}
\hline General & REP & T. $^{\text {a }}$ de la Ed. & I\&A & Psicodidáctica & Comunicar \\
\hline $13,8 \%$ & $5,66 \%$ & $5,59 \%$ & $12,09 \%$ & $7,81 \%$ & $26,06 \%$ \\
\hline
\end{tabular}

Como ya adelantamos en los resultados referidos a los datos generales, el porcentaje medio de autores extranjeros en las 5 revistas se sitúa en el 13,8\%, y la revista con mayor presencia de investigadores foráneos es Comunicar: Revista Científica de Comunicación y Educación seguida de Infancia y Aprendizaje. Ambas revistas señalan explícitamente su carácter internacional al apuntar su pretensión de difusión en el ámbito iberoamericano. Infancia y Aprendizaje incluye en su Equipo Editorial un órgano de Editores Asociados en Latinoamérica, lo que hace prever la intención de difundir su trabajo en dicho contexto. Tanto es así que el 60\% de las publicaciones extranjeras son iberoamericanas. Comunicar: Revista Científica de Comunicación y Educación por su parte es bilingüe en todos sus artículos y dice tener vocación latinoamericana o ser una revista de ámbito latinoamericano. De 173 participaciones de centros extranjeros de hecho el 75,14\% son instituciones iberoamericanas.

De forma similar al apartado anterior, acudimos a los criterios de calidad científica establecidos por la FECYT (2007) para valorar el grado de internacionalización de las revistas, según el cual más del 15\% de los autores deben tener procedencia extranjera. Puesto que con los datos proporcionados por las revistas resulta imposible conocer la nacionalidad de los investigadores, se calcula este criterio mediante el origen de la institución a la que pertenecen. Así, sólo Comunicar: Revista Científica de Comunicación y Educación cumpliría el criterio de calidad relativo a la internacionalización al rozar el 20\% de autores de procedencia extranjera.

Otro aspecto interesante a analizar aquí es la evolución de los autores de procedencia extranjera durante el periodo analizado, prestando especial atención a la entrada de las revistas en el JCR. Esto a priori supondría una exposición de las revistas en el escaparate internacional, que conllevaría una atracción de autores de otros países.

TABla 25. Evolución de los AUTORES DE PROCEDENCIA EXTRANJERA

\begin{tabular}{|c|c|c|c|c|c|c|c|c|c|}
\hline 2001 & 2002 & 2003 & 2004 & 2005 & 2006 & 2007 & 2008 & 2009 & 2010 \\
\hline 15 & 27 & 17 & 28 & 38 & 31 & 33 & 33 & 41 & 64 \\
\hline $7,2 \%$ & $12,1 \%$ & $7,8 \%$ & $13,8 \%$ & $18,4 \%$ & $13,9 \%$ & $14,9 \%$ & $13 \%$ & $13,9 \%$ & $20,1 \%$ \\
\hline
\end{tabular}


GrÁFico 8. EVOlución DE lOS Autores DE PROCEDENCIA EXTRANJERA

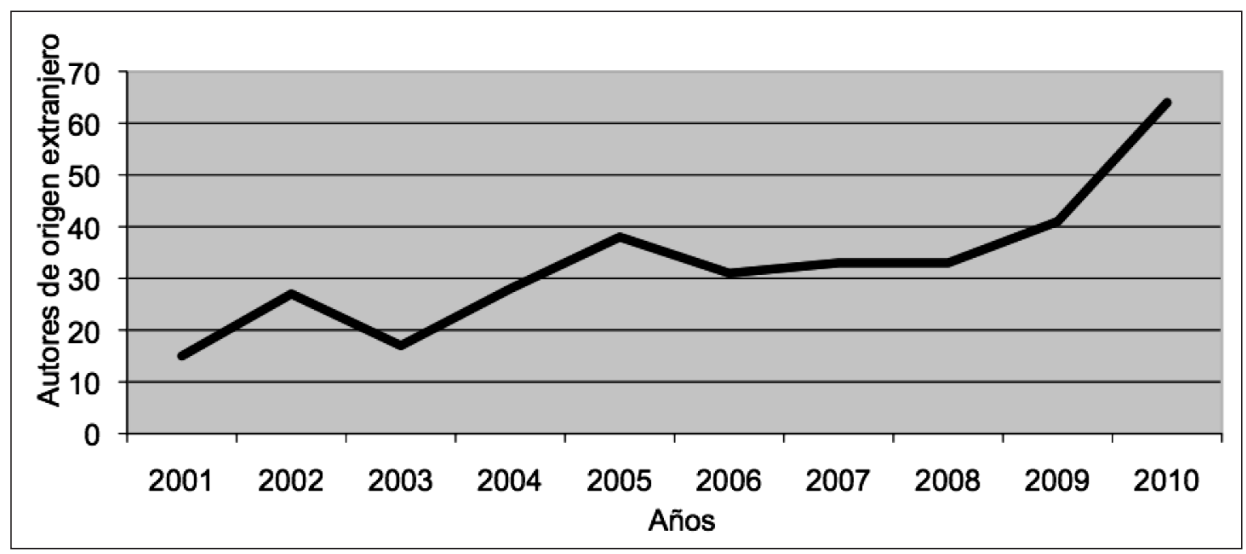

Así pues, a diferencia de lo que ocurría en los datos generales, se observa un incremento significativo de autores de procedencia extranjera en el periodo estudiado, multiplicando por tres el número de autores con respecto al primer año y aumentando en 13 puntos porcentuales. Además, este crecimiento se produce especialmente en los últimos años en los que las revistas entran en JCR y, muy importante, en 2010 donde todas se encuentran incorporadas en este índice.

A continuación, se muestran los autores y universidades de procedencia extranjera que más publican:

TABla 26. AuTORES y unIVERSIDADES DE PROCEDENCIA EXTRANJERA MÁS PROLÍFICOS

\begin{tabular}{|c|c|c|c|}
\hline Autores & $\begin{array}{c}\mathrm{N}^{\circ} \text { de } \\
\text { artículos }\end{array}$ & $\begin{array}{c}\text { Miembro del } \\
\text { organigrama científico }\end{array}$ & $\begin{array}{c}\text { Idioma de } \\
\text { publicación }\end{array}$ \\
\hline $\begin{array}{c}\text { Gómez, Juan Carlos } \\
\text { (Saint Andrews University) }\end{array}$ & 3 & Infancia y Aprendizaje & Inglés-español \\
\hline $\begin{array}{c}\text { Fontcuberta, Mar } \\
\text { (Universidad Pontificia Católica de Chile) }\end{array}$ & 3 & Comunicar & Español \\
\hline $\begin{array}{c}\text { Reia Baptista, Vitor } \\
\text { (Universidad del Algarve) }\end{array}$ & 3 & Comunicar & Portugués \\
\hline $\begin{array}{c}\text { Contin, Silvia Andrea } \\
\text { (Instituto Superior de Formación } \\
\text { Docente. Argentina). }\end{array}$ & 3 & Comunicar & Español \\
\hline
\end{tabular}


JUAN LUIS FUENTES, DAVID LUQUE Y ERNESTO LÓPEZ GÓMEZ

ANÁLISIS BIBLIOMÉTRICO DE LAS REVISTAS ESPAÑOLAS DE EDUCACIÓN

Entre las universidades extranjeras con mayor presencia están las que se muestran en la siguiente tabla:

\section{TABLA 27. UNIVERSIDADES EXTRANJERAS CON MAYOR PRESENCIA EN LAS REVISTAS ESPAÑOLAS}

\begin{tabular}{|l|c|c|}
\hline \multicolumn{1}{|c|}{ Instituciones } & \multicolumn{2}{c|}{ Participación en artículos } \\
\hline Universidad Nacional Autónoma de México & 16 & $4,9 \%$ \\
\hline Universidad de Minho & 14 & $4,3 \%$ \\
\hline Universidad Virtual Monterrey & 7 & $2,1 \%$ \\
\hline Universidad Pontificia Católica de Chile & 7 & $2,1 \%$ \\
\hline Universidad de Buenos Aires & 7 & $2,1 \%$ \\
\hline Universidad Nacional de La Plata & 6 & $2,1 \%$ \\
\hline Universidad de los Andes & 6 & $1,8 \%$ \\
\hline Universidad de Guadalajara & 6 & $1,8 \%$ \\
\hline Universidad de São Paulo & 5 & $1,8 \%$ \\
\hline Universidad Distrital Francisco José de Caldas & 5 & $1,5 \%$ \\
\hline Universidad Nacional de Colombia & 5 & $1,5 \%$ \\
\hline Universidad de Porto & 5 & $1,5 \%$ \\
\hline
\end{tabular}

\subsubsection{Idiomas preferidos de publicación}

Si atendemos al idioma preferido de publicación se observa que sólo el 4,64\% se publica en un idioma diferente al español, el 3,73\% si restamos las publicaciones en vasco, que suponen la cuarta lengua más elegida y que al ser lengua cooficial no cabe situarla en el apartado de la internacionalización.

El desglose de estos datos por revistas nos da alguna información sobre la orientación de las mismas. En la Revista Española de Pedagogía se publica casi totalmente en español: 4 en inglés de 244 artículos. En Teoría de la Educación. Revista Interuniversitaria se mantiene la misma línea aunque se incluyen artículos de otros idiomas: 2 inglés, 1 portugués y 3 italiano de 105. Infancia y Aprendizaje presenta porcentajes similares a Teoría de la Educación. Revista Interuniversitaria, pero sólo publica artículos en inglés: 14 de 300. Revista de Psicodidáctica es donde mayor porcentaje de otros idiomas hay y donde se publican los artículos en vasco, algo coherente pues es editada por la Universidad de País Vasco y, como se vio, la mayor parte de los autores que publican en ella son de esta universidad. Sin embargo, si se suma el vasco y el español, los porcentajes de artículos de otros países son muy similares al resto de revistas. En Comunicar: Revista

5. Este porcentaje se ha obtenido de la participación de las universidades extranjeras más representativas. 
Científica de Comunicación y Educación, de forma similar a la Revista Española de Pedagogía, casi la totalidad de los artículos están en español. Cabe destacar que todos los publicados en otra lengua son en portugués, lo que va en coherencia con el carácter iberoamericano de esta revista. Sin embargo, su presencia es meramente testimonial: 12 de 502. La conclusión que se puede extraer de este apartado es la tendencia general a publicar artículos en español casi en su totalidad, con algunas pequeñas excepciones.

\subsubsection{Traducciones}

Otro criterio que puede servir para evaluar la internacionalización de las revistas es el número de artículos traducidos al español. Así, 40 artículos son traducciones, a saber, un 3,04\%, lo que junto con el dato anterior nos indica que el 7,68\% de los artículos están escritos por autores que no hablan español ${ }^{6}$.

\section{DisCUSIÓN}

Los datos obtenidos son desde luego lo suficientemente esclarecedores como para poder pensar críticamente el estado de las revistas de investigación educativa en España incluidas en el $J C R$, así como el estado de las investigaciones educativas en nuestro país.

Para empezar, cabe señalar que la evolución en el número de artículos publicados no tiene por qué aportar información sobre el mayor o menor número de trabajos enviados a las revistas para su evaluación. Como hemos visto, la evolución en el número de artículos publicados no trae consigo un crecimiento significativo ni durante el periodo de tiempo comprendido en la década analizada ni desde que cada revista fue incorporada en el JCR.

Cabría pensar que por supuesto al haber sido catalogadas en tan selecto índice el número de artículos enviados para ser publicados debería haber crecido, lo que se supone que debería haber supuesto un incremento en el número de artículos publicados. Pero los datos revelan que la realidad no es así. Ahora bien, ¿cómo puede explicarse este dato?

- Razones de naturaleza económica. En un primer momento cabría pensar que los recursos de financiación con que cuenta cada revista para editarse debieran aumentar con el número de artículos publicados y ante la imposibilidad de conseguir financiación más allá de los organismos que ya editan las revistas se ha optado por mantener el formato que tenían

6. No se incluyen aquí aquellos que se escriben en colaboración con un autor que sí lo habla; además hay algunos artículos, aunque muy pocos, escritos por españoles en inglés; también se incluyen aquí los escritos en vasco que es lengua cooficial y por lo tanto supone que se conozca también el español por sus autores. 
antes de ser indexadas. Esta razón, aunque plausible, es sin embargo difícil de mostrar por la imposibilidad de acceder a las cifras económicas que manejan los journals.

- Razones selectivas. Otra razón sería que el hecho de haber sido indexadas en el $J C R$, con el plus añadido que esto conlleva para la imagen de las revistas en cuestión, les ha podido conducir a una posición privilegiada en la que pueden permitirse el lujo de rechazar artículos que siendo de gran calidad no lo son de tanta como los que finalmente ven la luz en sus páginas ${ }^{7}$. Esto, que en un principio podría parecer negativo por el volumen de artículos que se rechaza, no lo es tanto si se tiene en cuenta que puede provocar un efecto de lo que podría llamarse "dispersión científica", esto es, los artículos rechazados de una cierta calidad que fueron escritos con la pretensión de ver la luz en las revistas del JCR van a otras revistas que no lo están y elevan así el nivel de éstas, lo que presumiblemente puede traer la consecuencia de que en años venideros cada vez más revistas serán indexadas en el JCR.

- Razones de política editorial. Dentro de este espacio se pueden encontrar dos perspectivas. Los diversos miembros del organigrama científico deciden seguir una tendencia continuista, esto es, aplicar el pensamiento de que si una cosa funciona no tiene sentido cambiarla, y menos aún si con lo realizado se ha conseguido entrar en el $J C R$. La otra perspectiva es que aumentar el número de artículos publicados conlleva por necesidad un aumento en el número de horas que hay que dedicar a la edición de cada número y/o un aumento de los miembros de los equipos evaluadores, lo que no tiene por qué traer siempre buenas consecuencias.

Otro dato llamativo es la escasa representatividad de instituciones de educación no superior en el porcentaje total de publicaciones, un escaso 2,9\%, y de otros organismos, 6,4\%, lo que puede interpretarse de dos formas. Por un lado, la investigación pedagógica ha quedado reducida al ámbito de la universidad. Este hecho, que puede ser interpretado positivamente desde un punto de vista clásico en el que los profesores de enseñanza primaria y secundaria se dedican a enseñar y los profesores universitarios además a investigar, es negativo desde el paradigma de la investigación-acción en educación cuyo ideal no parece alcanzar el éxito esperado al menos en las revistas del $J C R$, quizá porque la formación para la investigación en un mundo cada vez más especializado requiere de una formación

7. Este ejemplo de selectividad puede ejemplificarse perfectamente en la información que la revista Teoría de la Educación incluye en la "Memoria Anual" colgada en su página web: "De los 35 trabajos recibidos 15 han sido publicados, 16 han sido rechazados y otros 4 están pendientes de publicación. La tasa de rechazo resultante es del 45,70\%". Ver: http://campus.usal.es/ teoriadelaeducacion/memoriaanual. html [consultado el 08.07.2011.] Asimismo, la propia FECYT $(2007,75)$ identifica como criterio de calidad la tasa de aceptación de trabajos, que sitúa en un porcentaje de igual o inferior al 60\%. 
específica que los maestros no reciben, pues ya tienen bastante con dar respuesta a los desafíos curriculares del momento. La otra perspectiva es que la lejanía que evidencian estos datos entre el ámbito de la investigación y el de la realidad educativa bien podría traducirse también en una preocupante distancia entre las investigaciones pedagógicas y las aulas sobre las que éstas reflexionan, cuando, como dice Fernando Gil Cantero, la filosofía de la educación es una filosofía inevitablemente práctica que por necesidad se dirige a mejorar la práctica educativa (Gil Cantero, 2003).

El tercer dato a destacar es el grado de colaboración de los autores donde, aunque encontramos una media general cercana al 2 en número de investigadores por artículo, los trabajos individuales siguen primando sobre los colectivos en la mayoría de las revistas. La valoración de estos resultados es una cuestión discutible, pues aunque desde instancias internacionales se aboga por promover la investigación colaborativa y algunos autores consideran este grado de colaboración como bajo e individualista ( vid. Fernández Cano, 2011, 435), la metodología utilizada depende en gran medida del objeto de estudio, de tal forma que si bien algunas materias son más propicias para la participación de varios investigadores, en otras resultan necesarios el análisis y la reflexión individual. Este criterio no parece seguirse por las instituciones responsables de la financiación de la investigación tal y como se muestra en los datos encontrados, pues tienden a apoyar el trabajo colectivo frente al individual.

Así pues, los datos encontrados pueden valorarse de dos maneras. Por un lado, mediante el estudio de la evolución en el tiempo que, como ya constatamos, muestra un aumento muy importante en un periodo cuyo fin coincide con el del plazo proporcionado a los Estados europeos para la adaptación de sus sistemas universitarios al EEEs, en el que, como ya señalamos, se concede especial relevancia a la realización de trabajos de investigación en colaboración. Y, por otro, a través de la comparación con otros estudios similares que proporcionan datos sobre revistas del ámbito educativo. De esta forma, puede decirse que el grado de coautoría obtenido es superior al mostrado en la Revista Complutense de Educación $(1,3)$ (Carpallo y Burgos, 2009) pero similar al de Bordón (1,7) (Zych, 2011). Asimismo, el porcentaje de artículos colectivos es superior en el conjunto de nuestras revistas $(44,21 \%)$ que en las 2 publicaciones citadas (23,21\% y 42\%).

En cuanto a los datos que nos ofrece la endogamia, cabría hacer algunas apreciaciones previas que sirvan para situar la discusión en este punto. Estas consideraciones previas se reducen a que la endogamia puede ser vista como una práctica positiva o negativa, dependiendo del nivel con el que nos encontremos.

Si bien es cierto que esta práctica podría considerarse positiva al entender que los especialistas tienden a publicar en journals relacionados con su especialidad no lo es menos que es por lo general una práctica perjudicial para el buen estado de salud de la comunidad científica. Es razonable pensar que por supuesto la aspiración de los investigadores no debe reducirse a difundir sus descubrimientos científicos exclusivamente entre los colegas con los que comparten lugar de trabajo. Si fuera así poco o nada se habría adelantado respecto al siglo XVII y podríamos volver 
tranquilamente a la correspondencia privada o las publicaciones locales para difundir los hallazgos científicos. La pretensión de universalidad -tal como y como se recoge en la Carta Magna de las Universidades Europeas (1988)-, muy al contrario conduce a que las instituciones de educación superior que aspiran a un cierto grado de excelencia impidan estas prácticas porque si no lo hicieran nos encontraríamos con investigadores encerrados en torres de marfil que comparten sus descubrimientos únicamente con un reducido e invariable número de colegas.

Por otro lado, debido al aún reducido número de opciones para publicar en alguna de las revistas incluidas en el JCR de habla hispana, el criterio endogámico debe ser relativizado, pues no deben despreciarse los deseos de un investigador por publicar en una de las mejores revistas de su área en su país y en su lengua, exclusivamente porque esté dirigida por él mismo o por un compañero de despacho, departamento o universidad. Aun más, sabiendo de las dificultades y de los altos requerimientos para conseguir que una revista sea aceptada en las bases de datos más prestigiosas, no sería justo minusvalorar el trabajo de quienes además de llevar a esa revista a lo más alto siendo parte de su organigrama, presentan trabajos en la misma, que siendo evaluados por pares en doble ciego, son aceptados para su publicación. Y por último cabe añadir otro elemento más, pues los mayores especialistas en un área de conocimiento suelen ser llamados a formar parte de los consejos de las revistas de su área, y en algunos casos, no sólo de una de ellas, sino de varias. Esto ocurre en las revistas españolas de educación, en cuyos consejos pueden encontrarse profesores que son también parte del consejo de otra revista.

Dicho lo cual, no puede decirse que las revistas indexadas en el JCR sean por lo general cauce de expresión para los miembros del organigrama científico de las respectivas revistas, pues un 14,91\% según el criterio de la FECYT es alto, pero no lo suficiente como para afirmar que el nivel endogámico es negativo. Esto se puede interpretar como una declaración de intenciones que demuestra un mayor interés por difundir los trabajos de calidad de personas ajenas a la revista que por sus propios trabajos y cuando hacen esto último es de presuponer que lo hacen también bajo premisas de calidad. No obstante, hay excepciones en este sentido que consideramos honrado comentar en favor de revistas que presentan un muy bajo índice de endogamia editorial. Los casos de las revistas Teoría de la Educación. Revista Interuniversitaria, con un 20,99\%, e Infancia y Aprendizaje con un $17,15 \%$, elevan el porcentaje general si consideramos que la tercera revista en este porcentaje está a 10 puntos porcentuales de diferencia de la segunda.

Por otro lado, la endogamia universitaria nos permite una visión más ajustada de las dos revistas editadas por instituciones de educación superior. En el caso de Teoría de la Educación. Revista Interuniversitaria, observamos que no existe una alta representatividad en las publicaciones de la universidad editora, mientras que en Revista de Psicodidáctica, aunque presentaba porcentajes muy bajos en cuanto a la endogamia editorial, los datos se disparan en lo que respecta a la universitaria, donde un 45,55\% de sus artículos se vinculan a la universidad editora de la revista (Tabla 20). 
Cabría señalarse que los datos arrojados, dentro de la diversidad de cada revista, podrían mejorarse sustancialmente gracias a la entrada de nuevas publicaciones periódicas en el JCR en las categorías educativas. Así, los investigadores tendrán oportunidad y posibilidad de publicar en un amplio abanico de revistas lo que podría revertir en una reducción directa de los niveles de endogamia.

En cuando a las investigaciones financiadas, se observaba una tendencia al alza a medida que las revistas o bien entraban o bien se afianzaban en el JCR, aspecto que tiene su interés pues aunque no hay relación aparente entre los recursos y eficiencia en la investigación, una inyección económica sí sirve para desarrollar grandes líneas de investigación y contribuir al nacimiento o consolidación de grupos de investigación. Dicho lo cual, parece prudente hacer notar que dentro de esta tendencia al alza los inversores optan por financiar estudios dentro de la categoría de "Psicología de la Educación", lo cual puede interpretarse de distintas maneras. En efecto, esta tendencia nos muestra que se invierte posiblemente en un ámbito investigador de talante empírico, que ofrece soluciones prácticas a los problemas educativos emergentes. Y bien podría esto interpretarse como la fluctuación que se vive también en la política educativa: se buscan recetas que ayuden a elevar los resultados educativos de una forma rápida y eficiente. La otra perspectiva desde la que puede leerse esta tendencia para la discusión es que quizá la reflexión procedente de la filosofía y la teoría de la educación no consiguen de forma rápida resultados prácticos eficientes o bien que hay una gran distancia entre las reflexiones especulativas y el público interesado en el día a día de la educación.

El grado de internacionalización de nuestras revistas puede leerse en clave europea, esto es, desde la pretensión del EEEs de crear un marco donde el intercambio de estudiantes y conocimientos haga de las universidades europeas un destino atractivo para los mayores intelectuales, o en clave muchísimo más amplia, transoceánica, donde la puerta se abra a autores de otros continentes. Sea como fuere, en nuestro estudio esto se refleja en la procedencia del autor o en el idioma en que los artículos en cuestión se publican en las revistas y, a la vista de los resultados, aunque en progresión positiva, a día de hoy no puede decirse que haya un grado de internacionalización elevado a nivel europeo, donde se ha adoptado el inglés como la primera lengua para el intercambio científico. No obstante, sí existe algo más a nivel transoceánico donde se aprecia un grado de internacionalización considerable con instituciones procedentes de Iberoamérica. Esto tiene únicamente una lectura posible: la gran barrera para la internacionalización de la investigación en España es el idioma. Que las revistas de mayor prestigio dentro de las fronteras nacionales publiquen en su mayoría artículos en castellano podría deberse a la pretensión de situar nuestra lengua entre una de las principales en el intercambio científico junto al inglés y eso es un punto a favor de la política que parecen haber adoptado en general las revistas españolas indexadas en el JCR. Pero por el contrario no las hace atractivas para el resto de investigadores europeos pues tan sólo un 1,22\% de las publicaciones es en inglés, lo que podía considerarse una excepción, e interpretarse definitivamente como que no son un destino atractivo 
para las principales investigaciones en esa lengua que se hace extensible también a los investigadores de los Estados Unidos de América. Aunque se viene hablando de un aumento en la internacionalización de la investigación educativa española en los últimos años (Aliaga y Correa, 2011) la aceptación de revistas educativas en índices internacionales de calidad como el JCR es requisito necesario pero no suficiente para llegar a esta conclusión (Zych, 2011). Para poder afirmar que se posee tal característica debería asumirse, entre otros aspectos, que nuestras publicaciones periódicas son un referente internacional para la publicación de autores de otros países distintos al nuestro. Es decir, que nuestras revistas no fueran solamente el medio de difusión de los investigadores españoles lo que podría llevarnos a asumir un nuevo criterio de "endogamia nacional".

\section{CONCLUSIONES}

Sin lugar a dudas, la progresiva incorporación de revistas españolas de naturaleza educativa en prestigiosos índices internacionales como el JCR debe ser motivo de satisfacción para la investigación pedagógica de nuestro país. No obstante, el trabajo no finaliza con este resultado, pues la excelencia, lejos de ser un estado, es un proceso que requiere de una evaluación continua seguida del empeño por mantener los niveles de calidad a los que debe aspirarse en la Universidad del tercer milenio.

Igualmente relevante nos parece indicar que la producción científica educativa española -de calidad- no se reduce a su expresión en el JCR. No son pocas las revistas educativas nacionales que atienden a diversos criterios de calidad y que dan difusión a trabajos significativos para la investigación educativa o más concretamente para determinadas áreas para las que algunas revistas, pese a no estar en el JCR, son auténticos referentes. Encontramos, de este modo, que en el RECYT no solo se incluyen las revistas indexadas en las bases de Thomson Reuters sino que también aquellas que cumplen criterios de calidad ciertamente exigentes que evalúa la FECYT para tal efecto ${ }^{8}$.

De todos modos, el regocijo por las cinco primeras revistas aceptadas por este índice no debe ocultar la necesidad de prestar atención a algunos de los descubrimientos más significativos que nos deja el presente estudio:

1. La inclusión de las revistas en el JCR no implica una financiación extra por parte de ninguna institución, ni privada ni pública. Si desde el poder

8. Tras la resolución de la tercera convocatoria en marzo de 2012 quedan reconocidas con el sello de calidad FECYT las siguientes revistas: Bordón. Revista de Pedagogía, Revista de Investigación Educativa, Revista Española de Orientación y Psicopedagogía y Revista de Logopedia, Foniatría y Audiología que se unen a otras ya incluidas como Revista Complutense de Educación y Pedagogía Social: Revista Interuniversitaria. 
político se quiere invertir tanto en investigación y desarrollo como en educación, sería lógico pensar en financiar revistas que aumentan el prestigio de la investigación educativa a nivel internacional al ser reconocidas en índices de prestigio. Podría decirse sin embargo que se está más cerca de lo que sostiene Derek Bok (2003) que de cualquier otra cosa, a saber, que ese prestigio suele tener su origen en las investigaciones que reportan beneficios económicos y no en aquellas que incrementan la calidad educativa y docente.

2. Se tiende en exceso a financiar investigaciones del ámbito de psicología de la educación marginando en este sentido la reflexión procedente de la rama de la filosofía o de la teoría de la educación. Esta tendencia no es desde luego beneficiosa para la situación educativa pues el paso de los años y los resultados de los informes internacionales revelan que sin un marco teórico sólido, que suele provenir de la discusión filosófica de la educación, las novedades en educación son más similares a palos de ciego que a pasos firmes.

3. La adopción del español como idioma de publicación permite un gran intercambio científico con instituciones extranjeras de habla hispana, pero las aleja de ser un destino atractivo para investigadores de habla inglesa.

4. Se demanda una mayor apertura a investigadores ajenos a los organigramas científicos, universidades donde se editan las revistas y a otras instituciones de educación no superior.

\section{REFERENCIAS BIBLIOGRÁFICAS}

ALEIXANDRE, R. (2010a) La calidad de las revistas científicas: estrategias de mejora, en VALENCIANO, J. y Devís, J. (coords.) La calidad y evaluación de las revistas científicas a debate. Una mirada desde las Ciencias de la Actividad Física y el Deporte. Valencia, Puv, 101-114.

- (2010b) Bibliometría e indicadores de actividad científica, en JimÉnEz Villa, J.; ARGIMON, J. M.; Martín Zurro, A. y Vilardell, M. (eds.) Publicación científica biomédica. Barcelona, Elsevier España, 363-385.

Aleixandre, R.; Valderrama-Zurián, J. C. y GonZález Alcaide, G. (2007) El factor de impacto de las revistas científicas: limitaciones e indicadores alternativos. El Profesional de la Información, 16 (1), 4-11.

Aliaga, F. M. y CorreA, A. D. (2011) Tendencias en la normalización de nombres de autores en publicaciones científicas. Relieve, 17 (1), 1-10.

Apodaca. P. (2006) Estudio y trabajo en grupo, en De Miguel, M. (coord.) Metodología de enseñanzas y aprendizaje para el desarrollo de competencias: orientaciones para el profesorado universitario ante el Espacio Europeo de Educación Superior. Madrid, Alianza, 169-190.

ARgǘlLes, J. C. (2008) ¿Qué es la producción científica? El País, 2 de febrero.

Bellés, X. (2006) Cantidad y calidad de los artículos científicos. El País, 25 de octubre.

BOK, D. (2003) Universities in the Marketplace: The Commercialization of Higher Education. New Jersey, Princeton University Press. 
Bracho-LóPez, R.; Maz-Machado, A.; Gutiérrez-Arenas, P.; Torralbo-Rodríguez, M. e Hidalgo-ARIZA, M. ${ }^{a}$ D. (2010) Análisis temático de la investigación en educación matemática en revistas científicas españolas (1999-2008), en CASTRO, Á. y GuILLÉN Riquelme, A. (comps.) VII Foro sobre Evaluación de la Calidad de la Investigación y de la Educación Superior. Granada, Asociación Española de Psicología Conductual (AEPC). 359-363. Consultado el 11 de junio de 2011. http://www.ugr.es/ aepc/VIIFORO/VIIForoLibrocapitulos.pdf.

Carpallo, A. y Burgos, E. (2009) Estudio bibliométrico y de calidad de la Revista Complutense de Educación (1990-2007) Revista Complutense de Educación, 19 (1), 13-29.

Delgado López-Cozar, E. (2007) Índice de impacto de las revistas españolas de biblioteconomía y documentación. Anuario ThinkEPI, 32-42.

Delgado LóPEZ-COZAR, E. et al. (2005) IN-RECS: Índice de impacto de las revistas españolas de ciencias sociales. Una nueva herramienta para medir el impacto de la investigación española. Revista Bibliográfica de Geografía y Ciencias Sociales, 10 (574). Consultado el 11 de junio de 2011. http://www.ub.es/geocrit/b3w-574.htm.

FECYT (2007) La Edición de Revistas Científicas: Directrices, Criterios y Modelos de Evaluación. Madrid, FECYT.

FernáNDez CANO, A. (1999) Producción educativa española en el Social Sciences Citation Index (1988-1997). Revista Española de Pedagogía, 57 (214), 509-523.

- (2011) Producción educativa española en el Social Sciences Citation Index (1998-2009) II. Revista Española de Pedagogía, 69 (250), 427-444.

Flores Buils, R.; Gil Beltrán, J. M.; Caballer, A. y Martínez Martínez, M. A. (2012) La orientación profesional a través de Bordón. Revista de Pedagogía: Estudio cientimétrico. Bordón, 64 (1), 75-88.

FundACIÓN SANTA MARÍA (1994) La educación en la España Contemporánea (1789-1975). Madrid, Ediciones SM.

Galino, M. ${ }^{a}$ Á. (2005) Vivencias y datos para la reflexión. Centenario de los estudios de pedagogía en la Universidad, en Ruiz Berrio, J. y VÁzquez Gómez, G. (coords.) Pedagogía y educación ante el s. XXI. Madrid, Departamento de Teoría e Historia de la Educación. Universidad Complutense de Madrid, 15-36.

García Sánchez, J.; SÁnchez Miguel, E.; Arias-Gundín, O. y Del Río, P. (2002) Análisis de contenido de los 25 años de Infancia y Aprendizaje: historia de la investigación sobre desarrollo y educación. Infancia y Aprendizaje, 25 (4), 403-440.

GARFIELD, E. (1979) Citation indexing. Its theory and application in science, technology and bumanities. Nueva York, John Wiley and Sons.

Gil Cantero, F. (2003) La filosofía de la educación como teoría ética de la formación humana. Revista Española de Pedagogía, 61 (224), 115-130.

Gómez-García, A.; Ramiro, M. T.; Ariza, T. y Granados, M. R. (2012) Estudio bibliométrico de Educación XX1. Educación XX1, 15.1, 17-41.

Granados, M. R.; Ariza, T.; Gómez-García, A. y Ramiro, M. ${ }^{a}$ T. (2011) Estudio bibliométrico de Aula Abierta. Aula Abierta, 39 (3), 97-110.

Gutiérrez-Arenas, P.; MaZ-Machado, R. e Hidalgo-Ariza, M. ${ }^{a}$ D. (2010) Análisis de la coautoría en la revista Bordón (1984-2008), en CASTRO, A. y GuilléN-RiQuelme, A. (comps.) VII Foro sobre Evaluación de la Calidad de la Investigación y de la Educación Superior. Granada, Asociación Española de Psicología conductual, 749-753.

Hamilton, D. y Weiner, G. (2011) Dancing At The Edge: Writing For The Academic Marketplace. Education Inquiry, 2, (2), 251-262. 
Hernández DíAz, J. M. ${ }^{a}$ (ed.) (2010) Cien años de pedagogía en España. Valladolid, Castilla.

LEÓN DEL BARCO, B. y LATAS, C. (2007) La formación en técnicas de aprendizaje cooperativo del profesor universitario en el contexto de la convergencia europea. Revista de Psicodidáctica, 12 (2), 269-277.

PinedA, J. M. ${ }^{a}$ (1985) Historia de la educación. Estudio bibliométrico de las publicaciones periódicas en España (1949-1976). Historia de la Educación, 5, 423-430.

Ruiz Berrio, J. y VázQuez Gómez, G. (coords.) (2005) Pedagogía y educación ante el s. XXI. Madrid, Departamento de Teoría e Historia de la Educación. Universidad Complutense de Madrid.

Russell, J. M.; Madera-Jaramillo, M. J. y Ainsworth, S. (2009) El análisis de redes en el estudio de la colaboración científica. REDES-Revista Hispana para el análisis de redes sociales, 17 (2), 39-47.

Sánchez Miguel, E.; García Sánchez, J. y Del Río, P. (2002) Qué, cómo y cuándo: un análisis temático de lo publicado en los 25 años de Infancia y Aprendizaje. Infancia y Aprendizaje, 25 (4), 441-468.

Seglen, P. O. (1993) How representative is the journal impact factor? Research Evaluation, $2,143-149$

VÁzquez, G.; Colom, A. y Sarramona, J. (1998) Evaluación de la universidad. Criterios de calidad. Teoría de la Educación. Revista Interuniversitaria, 10, 55-94.

Vicentelli, H. (2009) Producción científica: Revista de Pedagogía de la Universidad Central de Venezuela (1971-2005). Revista de Pedagogía, 30 (86), 161-188.

ZYCH, I. (2011) Análisis bibliométrico de la Revista Bordón. Bordón, 63 (2), 141-152. 